ARTICLE

Corrected: Author correction

DOI: $10.1038 / s 41467-018-04678-8 \quad$ OPEN

\title{
A novel atypical sperm centriole is functional during human fertilization
}

Emily L. Fishman (10 ${ }^{1}$, Kyoung Joㄹ, Quynh P.H. Nguyen², Dong Kong (10 ${ }^{3}$, Rachel Royfman ${ }^{1}$, Anthony R. Cekic ${ }^{1}$, Sushil Khanal ${ }^{1}$, Ann L. Miller (10 ${ }^{4}$, Calvin Simerly ${ }^{5}$, Gerald Schatten ${ }^{5}$, Jadranka Loncarek ${ }^{3}$,

Vito Mennella ${ }^{2} \&$ Tomer Avidor-Reiss ${ }^{1}$

The inheritance of the centrosome during human fertilization remains mysterious. Here we show that the sperm centrosome contains, in addition to the known typical barrel-shaped centriole (the proximal centriole, PC), a surrounding matrix (pericentriolar material, PCM), and an atypical centriole (distal centriole, DC) composed of splayed microtubules surrounding previously undescribed rods of centriole luminal proteins. The sperm centrosome is remodeled by both reduction and enrichment of specific proteins and the formation of these rods during spermatogenesis. In vivo and in vitro investigations show that the flagellumattached, atypical DC is capable of recruiting PCM, forming a daughter centriole, and localizing to the spindle pole during mitosis. Altogether, we show that the DC is compositionally and structurally remodeled into an atypical centriole, which functions as the zygote's second centriole. These findings now provide novel avenues for diagnostics and therapeutic strategies for male infertility, and insights into early embryo developmental defects.

\footnotetext{
${ }^{1}$ Department of Biological Sciences, University of Toledo, 2801W. Bancroft, Toledo, OH 43607, USA. ${ }^{2}$ Cell Biology Program, The Hospital for Sick Children, Department of Biochemistry, University of Toronto, 555 University Avenue, Toronto, ON M5G 1X8, Canada. ${ }^{3}$ Laboratory of Protein Dynamics and Signaling, Center for Cancer Research, National Cancer Institute, 1050 Boyles Street, Frederick, MD 21702, USA. ${ }^{4}$ Department of Molecular, Cellular, and Developmental Biology, University of Michigan, 830 North University Ave, Ann Arbor, MI 48109, USA. 5 Departments of Cell Biology; Obstetrics, Gynecology and Reproductive Sciences; and Bioengineering, Magee-Womens Research Institute, University of Pittsburgh School of Medicine, 204 Craft Avenue, Pittsburgh, PA 15213, USA. Correspondence and requests for materials should be addressed to T.A-R. (email: Tomer.AvidorReiss@utoledo.edu)
} 
uman development begins with the zygote, which divides many times to produce all of the somatic cells. These somatic cells each contain two centrioles, which duplicate in a number-controlled manner from pre-existing centrioles. Since cells require two centrioles for normal division, one would expect the human zygote to have two centrioles during interphase and four centrioles during mitosis. However, four centrioles have never been shown in any mammalian zygotes; only three centrioles were observed ${ }^{1, ; 2}$. Since the human oocyte lacks centrioles, and the zygote's paternal pronucleus is associated with an aster of microtubules, it seems that the embryo's centrioles are paternally inherited $^{3,4}$. The paternal centrioles reside at the junction of the sperm nucleus and flagellum, in a region known as the neck. The neck region includes, in addition to the centrioles, the striated columns, and capitulum, which surround an electron-light region known as the vault ${ }^{5,6}$.

The current dogma is that the early sperm's centrioles and their surrounding pericentriolar material (PCM) are modified by a process called centrosome reduction during spermiogenesis $^{5,7}$. During reduction, the distal centriole's (DC) typical structure disintegrates, the proteins surrounding the centrioles that make up the PCM are eliminated, and a vault appears in the expected place of the $\mathrm{DC}^{8}$. Only residual microtubules and proteins are observed in the mature spermatozoa DC, and no function has been associated with the DC remnants or the vault ${ }^{6}$ (Fig. 1a). Therefore, the current prevailing dogma is that the sperm has a single functional centriole, the $\mathrm{PC}^{9-13}$, and therefore the zygote inherits only one centriole. Yet, the zygotic somehow provides four centrioles, two for each daughter cell. The inheritance of only a single centriole is problematic since centrioles form by duplication of pre-existing centrioles, which act as a platform for the formation of a single nascent daughter centriole. If the zygote inherits only one functional centriole, then the origin of the second interphase centriole is unknown.

A similar enigma existed previously in insects until it was shown that insect sperm possesses, in addition to the known centriole, a second atypical centriolar structure ${ }^{14-16}$. This atypical structure has been shown to be essential for normal fertility and embryo development ${ }^{15,17}$. The presence of an unexpected second centriolar structure in insects raises the possibility that humans possess a similar atypical structure that may be important during reproduction and development. Here we investigated human spermatozoa and bovine zygotes to determine if mammals have a functional atypical second centriole.

In this work, we examine several groups of centrosomal proteins, and find that a subset of them are present, unexpectedly in the DC of human spermatozoa. Using Correlative Light and Electron Microscopy as well as High Pressure Freezing, Freeze Substitution Electron Microscopy, we find that the DC is attached to the base of the axoneme, but that its microtubules splay outward, forming a novel, atypical, structure. Using Super-Resolution microscopy we find that the human and bovine DC's subset of centriolar proteins are organized into rods. During spermatogenesis in bovine testes, we find that the DC's rods appear during spermatid development. Next, we test the competency of the DC using an in vitro system and find that the human DC can recruit the PCM protein, $\gamma$-tubulin. Furthermore, we follow the DC of bovine sperm into the zygote and found that it recruits PCM, forms an aster, forms a new daughter centriole, and localizes to the spindle pole, all while maintaining its attachment to the axoneme. These findings discover a novel, atypical centriole in the sperm, which functions in the zygote.

\section{Results}

The DC contains a subset of centriolar proteins. The dogma of centrosome reduction and previously published literature states that PCM proteins are reduced based on the observations that $\gamma$ tubulin and PCNT are missing from the sperm centrosome ${ }^{5}$. Likewise, we found that the PCM proteins $\gamma$-tubulin and PCNT were eliminated from the neck region during spermatogenesis (Supplementary Fig. 1a). Only one protein thus far has been seen in the DC, CETN1/2; however, CETN1/2 was seen primarily in the PC, and only inconsistently in the DC, which was interpreted to be undergoing reduction ${ }^{5,6}$.

Contrary to this observation, we found that an antibody against the centriolar protein CETN1/2 equally labeled both the DC and PC in ejaculated human sperm (Supplementary Fig. 1a) ${ }^{5}$. Because of this unexpected observation of CETN1/2 in the DC, we sought to define the composition of the DC to determine whether it could be the second centriole of the spermatozoa. We identified candidate sperm centrosome proteins by comparing the spermatozoon proteome $e^{18-21}$ to the centrosome database ${ }^{22}$ (Supplementary Fig. 2, Supplementary Table 1). We examined the localization of candidates using immunostaining (Supplementary Fig. 1). Then, we validated the specificity of the antibodies in U2OS cells (Supplementary Fig. 3).

The putative centrosomal proteins were separated into five groups based on their localization in the spermatozoan neck region (Fig. 1b). Consistent with centrosome reduction, we found that several PCM/appendage/satellite proteins such as CEP152 and CEP192 were absent from the neck (Group i) (Supplementary Fig. 1b), and similarly some centriolar proteins such as CEP135 and CEP76 were seen only to associate with the intact PC (Group ii) (Supplementary Fig. 1c). Both of these groups support the idea that the some proteins are exclusively present in the PC and missing from the PCM the DC.

However, contrary to centrosome reduction, we observed several PCM/appendage proteins, such as CEP164 and CDK5RAP2, in the capitulum and striated columns (Group iii) (Supplementary Fig. 1d). These findings support structural studies showing that the capitulum and striated columns form in association with centriole microtubules, suggesting that the neck structures are a specialized form of $\mathrm{PCM}^{8}$. Also, contrary to centrosome reduction, we found some centriolar proteins such as CEP295 and CNTROB were absent from the PC and were either observed in the PCM or were undetectable, suggesting that the composition of the $\mathrm{PC}$ is also altered during centrosome reduction (Group iv) (Supplementary Fig. 1e).

Finally, several centriolar proteins labeled the DC (Group v). Of these proteins, we found that centriole distal lumen proteins CETN1/2 and POC $5^{23}$ localized equally to both the DC and PC (Fig. 1c). The PCM protein CEP63 and the centriole/PCM protein CPAP localized at the PC and DC (Fig. 1d). The transition zone protein, CEP290 24 , localized to the junction between the DC and the axoneme, presumably marking the tip of the DC (Fig. 1e). Interestingly, we found that a centriolar protein, $\mathrm{POC} 1 \mathrm{~B}$, which is enriched in the atypical centriole of Drosophila sperm $^{15}$, was similarly enriched in the DC relative to the PC. Three distinct antibodies confirmed that POC1B was enriched about two-fold in the DC in comparison to the PC (Fig. 1f). Altogether, these observations suggest that the DC is not eliminated during spermatogenesis but, instead, is present with an altered composition.

The DC is made of splayed microtubules. The POC1B enrichment of the DC was unexpected, and therefore we investigated the POC1B localization more precisely, using Correlative Light 
a

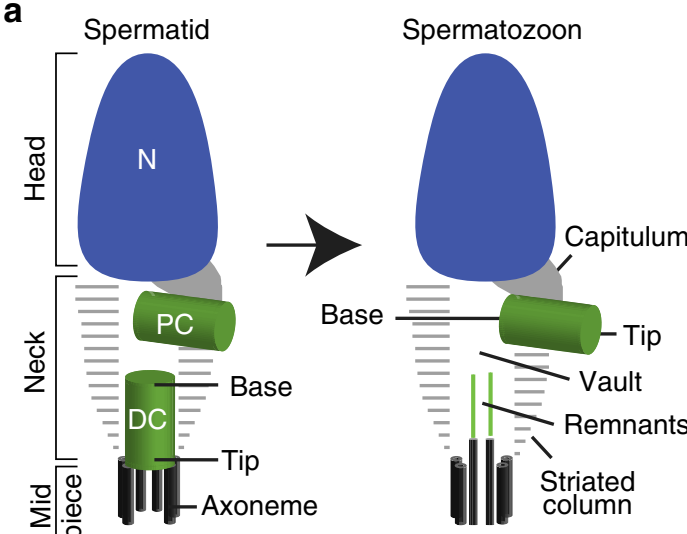

b

Consistent with the dogma

Group i

Absent PCM proteins

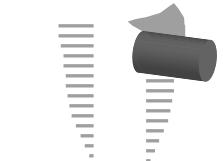

$\begin{array}{cc}\gamma \text {-tubulin } & \text { SSX2IP } \\ \text { PCNT } & \text { CEP89 } \\ \text { CEP192 } & \text { CEP41 }\end{array}$

CEP152

Inconsistent with the dogma

Group iii

Group iv

Present

Abnormally localised

PCM proteins centriole proteins
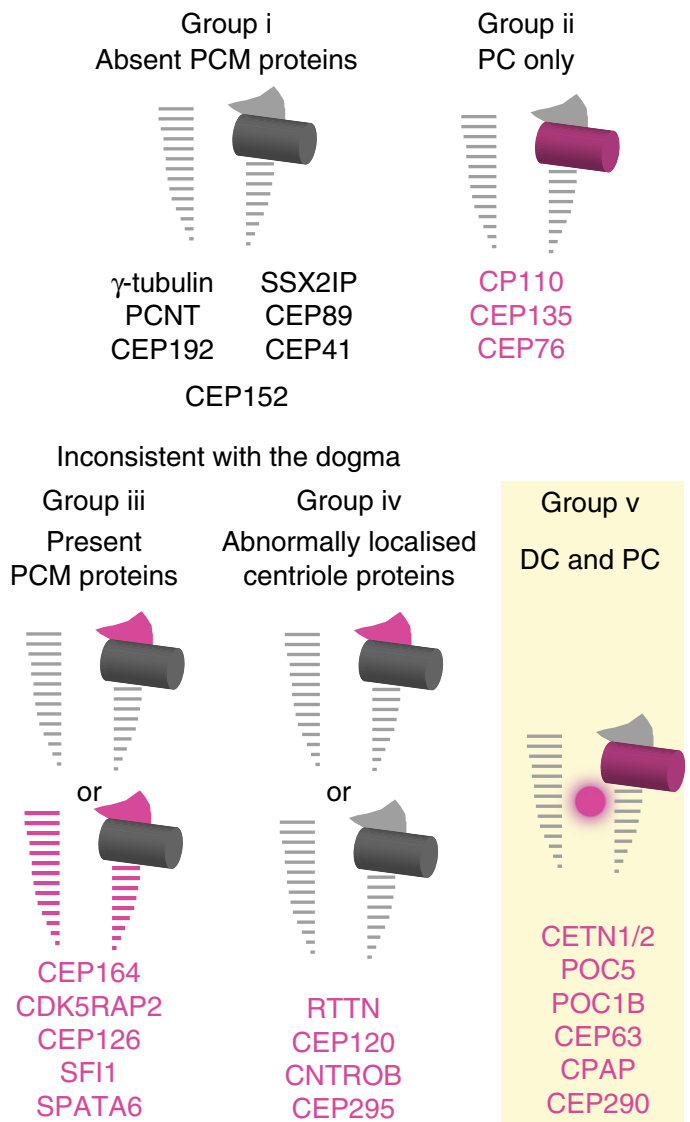

CETN1/2
POC5
POC1B
CEP63
CPAP
CEP290

c
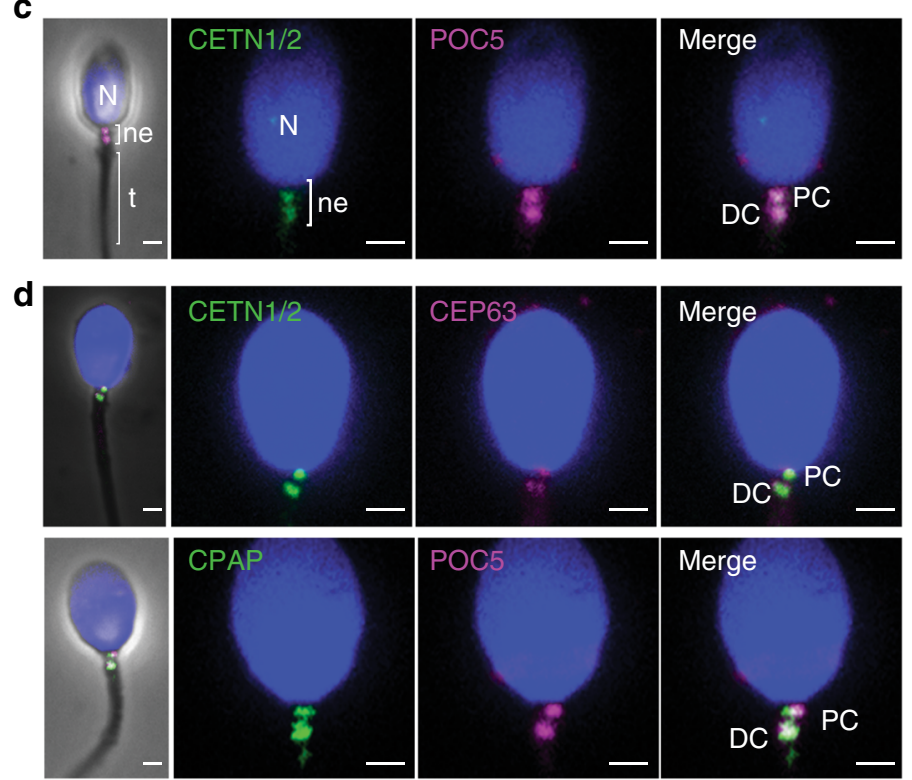

Merge

e
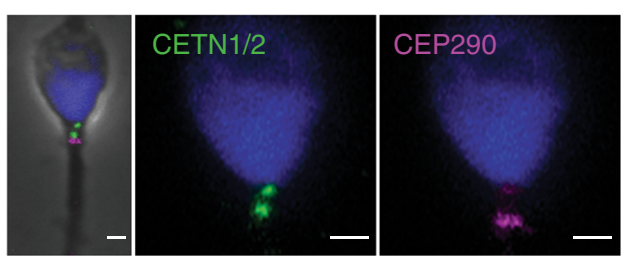

Merge

$\mathbf{f}$
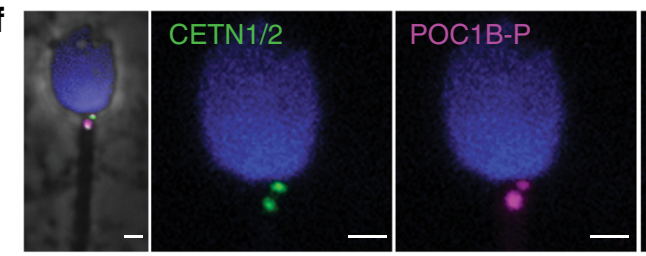

Merge
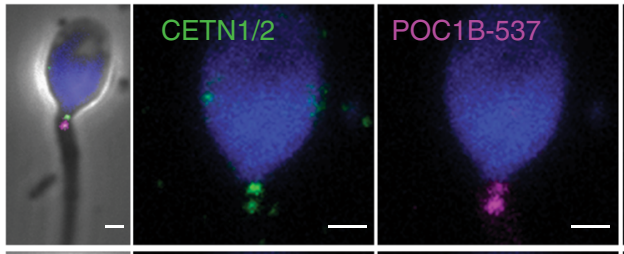

DC
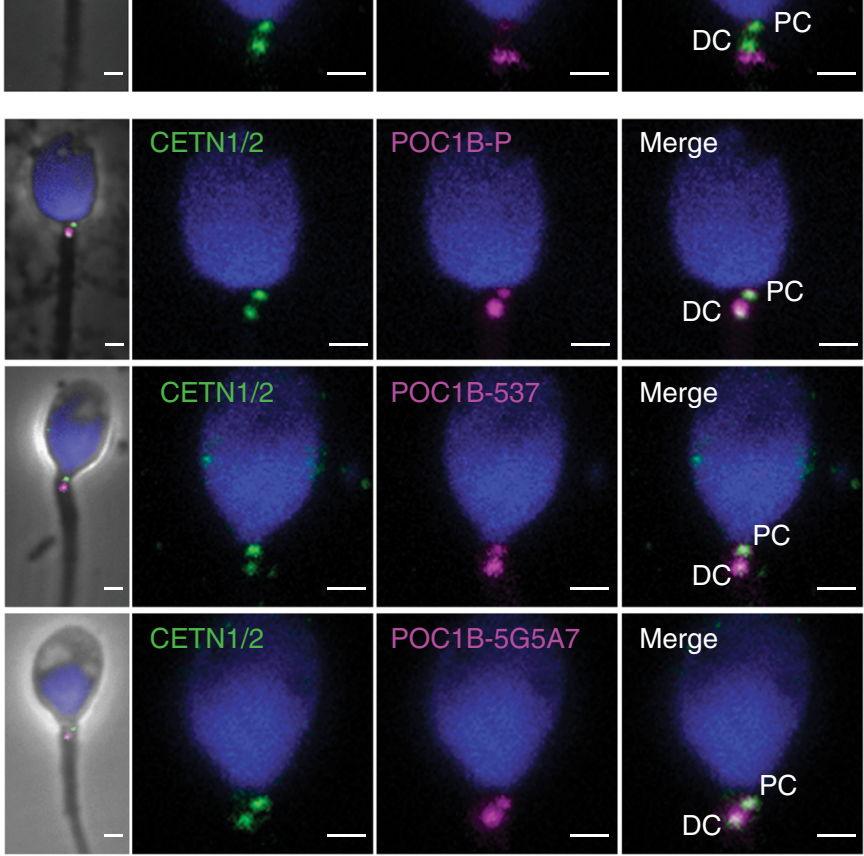

Fig. 1 Centriolar proteins localize to the DC (group v). a The current dogma is that mature sperm have one centriole. The spermatid neck has a PC and DC, and the ejaculated spermatozoon has a PC and an empty space, the vault, which marks the location of the degenerated DC (Manandhar et al. ${ }^{6}$ ). $\mathbf{b}$ Graphical summary of the groups of the localization patterns of proteins in the sperm. Group $\mathrm{i}$ is centriolar proteins that were localized to the PC area only and is consistent with the dogma. Group ii is PCM proteins that were absent from the sperm, as is consistent with the dogma. Group iii are PCM proteins that were unexpectedly localized to the striated columns or capitulum. Group iv are centriolar proteins that were unexpectedly localized to the striated columns or capitulum, or were unexpectedly absent altogether. Group $v$ are proteins that were unexpectedly present in the DC and the PC, which are inconsistent with the dogma. Group v is shown in c-f. c Antibodies against the centriole tip proteins CETN1/2 and POC5 labeled the PC and DC. d Antibodies against CEP63 and CPAP labeled near the CETN1/2 or POC5-labeled PC and DC. e Antibodies against CEP290 labeled adjacent to the CETN1/ 2-labeled DC, presumably marking the junction of the DC with the axoneme. $\mathbf{f}$ Three distinct antibodies against POC1B labeled both the PC and DC. Unlike other centriolar proteins, POC1B was enriched in the DC relative to the PC (POC1B-P: $2.1 \pm 0.5, P O C 1 B-537: 2.74 \pm 1.64, P O C 1 B-5 G 5 A 7: 1.92 \pm 0.41, N>6$ ). POC1B-P, Rabbit polyclonal antibody from the Pearson lab; POC1B-537, Rabbit polyclonal antibody from the Avidor-Reiss lab; POC1B-5G5A7 Rat monoclonal $\mathrm{Ab}$ from the Avidor-Reiss lab. $\mathrm{N}$ nucleus, ne sperm neck, $\mathrm{t}$ tail; scale bars $1 \mu \mathrm{m}$ 

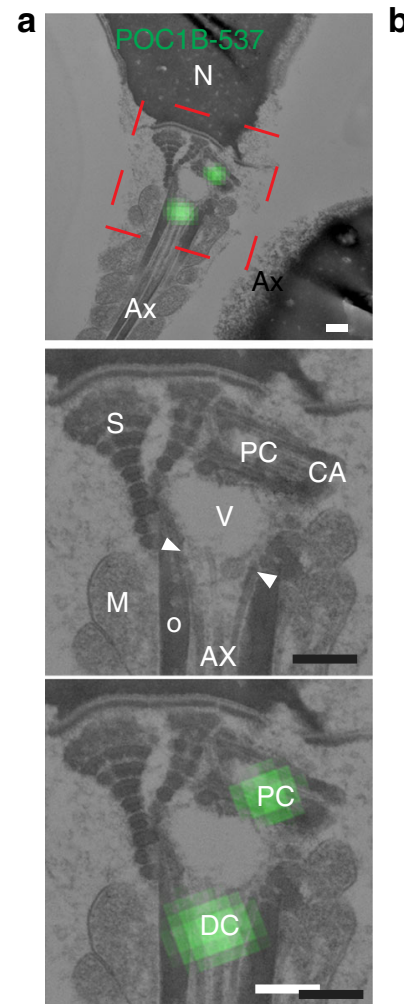

C
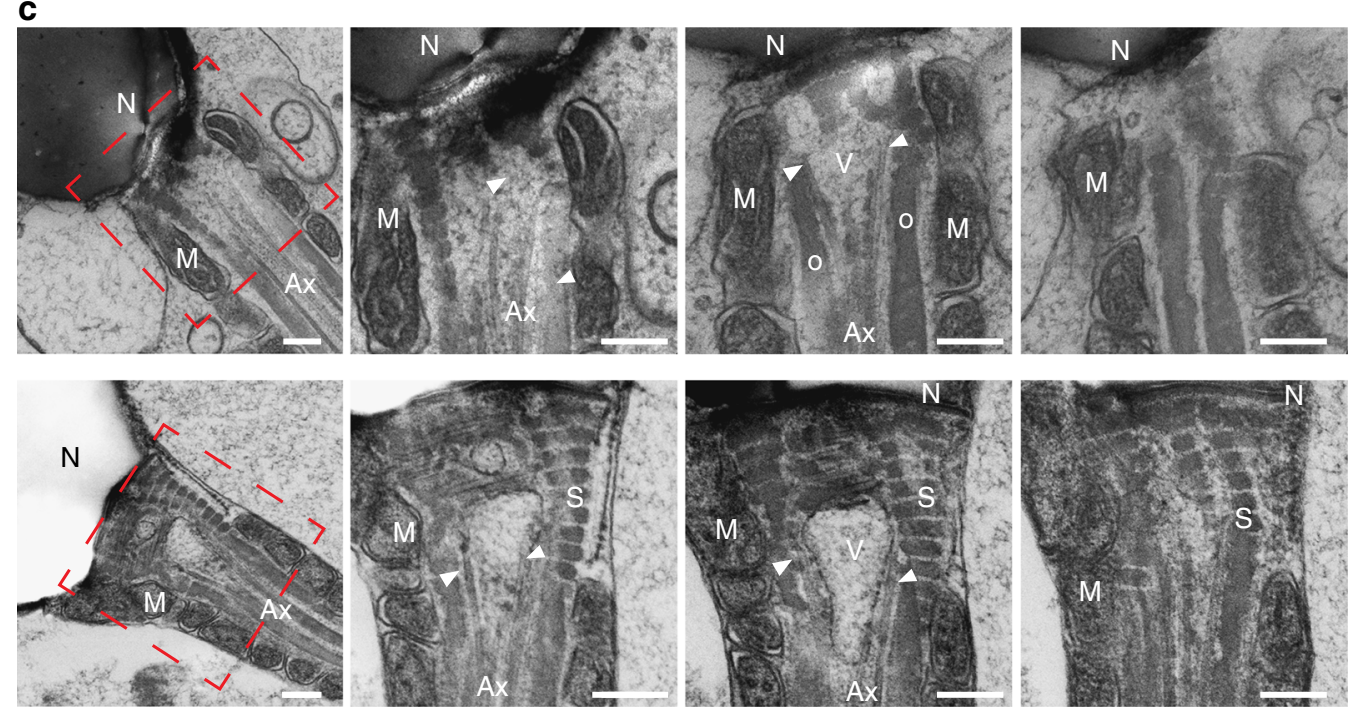

d
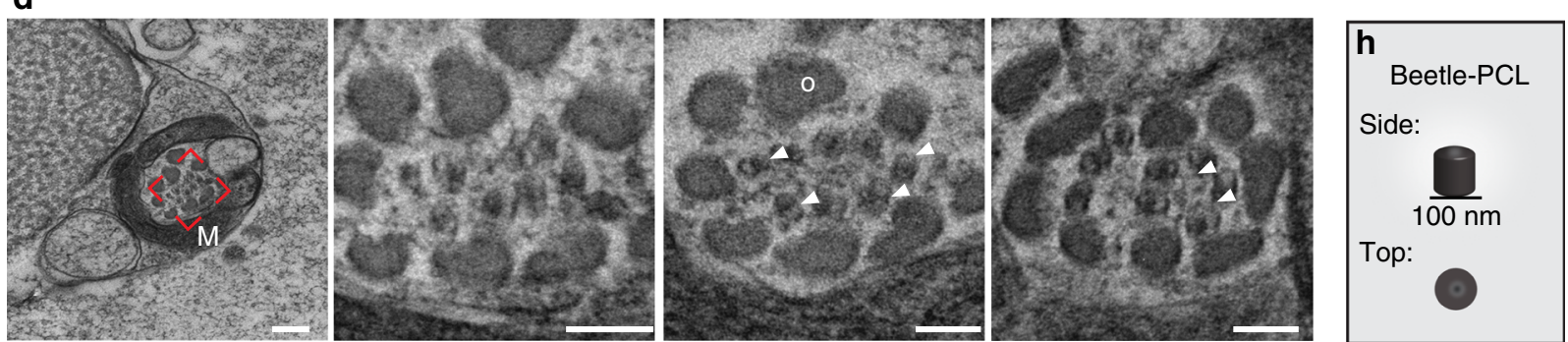

and Electron Microscopy. We found POC1B in the PC as well as at the base of the axoneme, which we interpret to be the DC (Fig. 2a). The DC is located between the vault and the axoneme, flanked by the outer dense fibers and the striated columns. This suggests that the DC is at the base of the axoneme, and the vault is located between the two centrioles.
To precisely identify the location of the DC's microtubules, we studied tubulin and POC1B, using four anti-tubulin antibodies (anti- $\beta$-tubulin E7, anti- $\alpha$-tubulin DM1A, anti-acetylated-tubulin, and sheep anti-tubulin). Each of the four anti-tubulin antibodies labeled two foci. Both of these foci colocalized with POC1B in the PC and the DC (Fig. 2b). In some cases, the tubulin signals in the 
Fig. 2 POC1B associates with splayed microtubules at the axoneme base. a The PC and the DC were labeled with POC1B using Correlative Light and Electron Microscopy. These structures were found to be associated with the splayed microtubules (arrowheads, between the axoneme and electron light vault). Scale bars $200 \mathrm{~nm}$. b Four distinct antibodies against tubulins labeled the DC and the PC with greater intensity than in the axoneme. Scale bars $1 \mu \mathrm{m}$. c, d Two examples of serial longitudinal sections showed splayed microtubules around an electron light vault using high-pressure freezing-freeze substitution of the sperm neck (c). Serial cross-sections showed splayed microtubules (d, arrowheads). Scale bars $200 \mathrm{~nm}$. e-h Models with side and top views of the human spermatozoon DC (e) and centriole (f), the fly spermatid's PCL (g), and beetle spermatid's PCL (h). Ax axoneme, M mitochondria, mp tail midpiece, $\mathrm{N}$ nucleus, ne neck, $\mathrm{O}$ outer dance fibers, pp tail principal piece, $\mathrm{S}$ striated columns, $\mathrm{V}$ vault

a

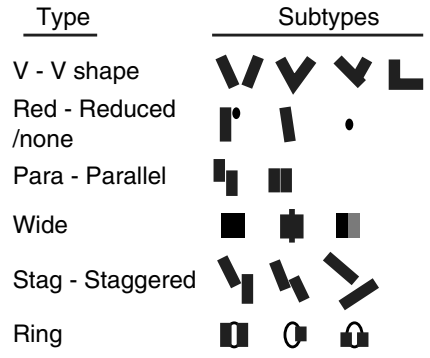

C
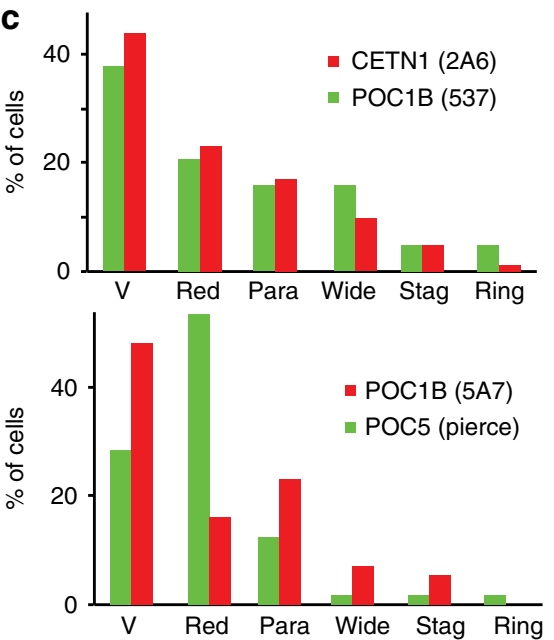

d

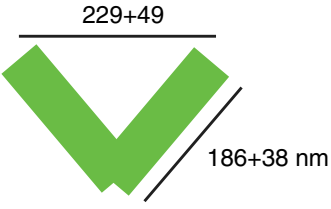

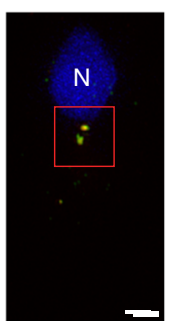
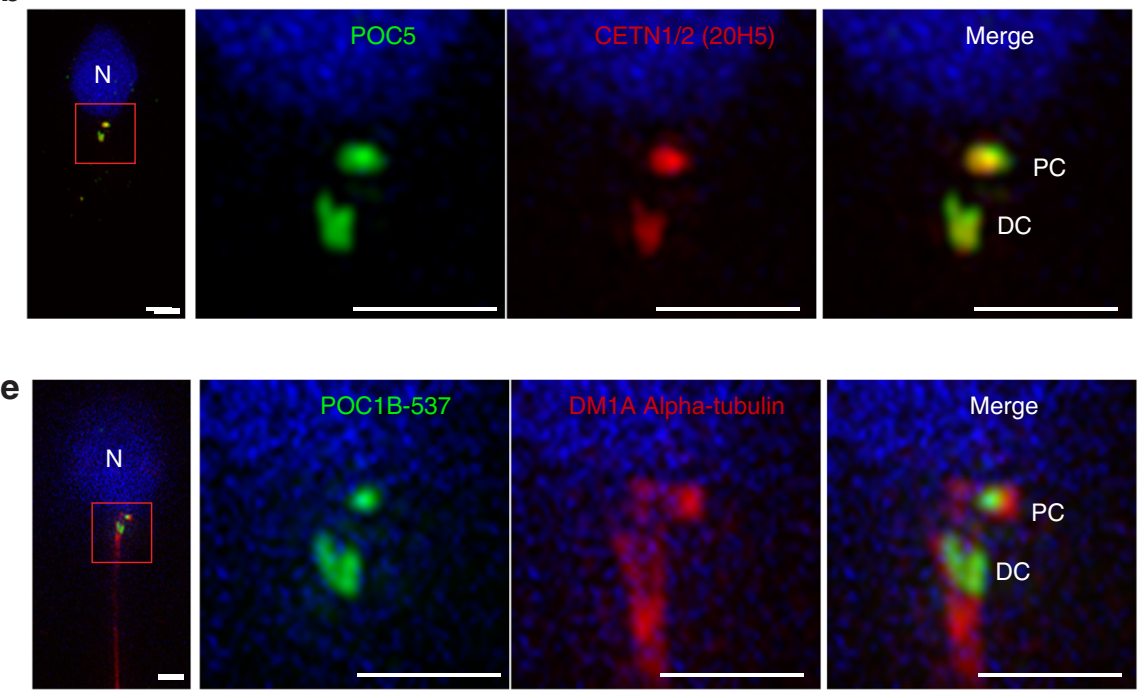

$\mathbf{f}$

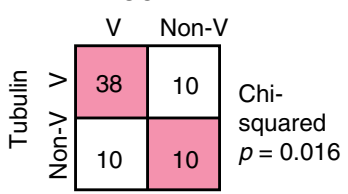

V shapes

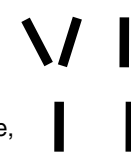

dot, line, none, $\Lambda$, ring)
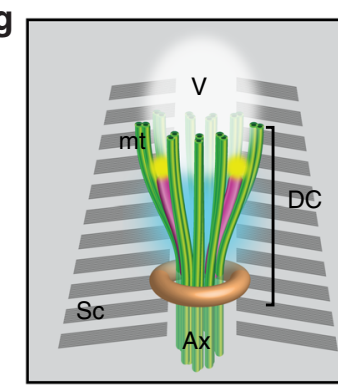

DC:

DC rods:

POC1B

CETN1/2

POC5
PC:

CEP76

CEP120

CEP135/BId10

POC1B

CETN1/2

POC5
PCM:

Striated columns:

RTTN

CEP164

CEP295

Capitulum:

CEP63

CDKRAP5/CEP215

Fig. 3 The precise location of DC proteins. a Diagrams of the six types of DC rods morphologies. $\mathbf{b}$ 3D-SIM showed "V"-shaped rods of centriolar proteins POC5 and CETN1/2. c Graph depicting the abundance of the six types of DC rods morphologies. The most common type for both CETN1/2 and POC1B is " $\mathrm{V}$ " shape (N 140). POC5 shows both a high rate of " $\mathrm{V}$ " shape ( 25\%), and a higher rate of "reduced" shape. $\mathbf{d} A$ diagram depicting the dimensions of the rods (height and width) and their distances from each other (from center to center of the rods) at their tips and bases ( $N \geq 20$ ). e $3 D-S I M$ showed " $V$ "shaped rods of centriolar protein POC1B flanking the splayed microtubules shown with DM1A, $\alpha$-tubulin (red). $\mathbf{f}$ Quantification of the relationship between POC1B shape and tubulin shape in 3D-SIM. Most cells that have "V"-shaped tubulin also have " $V$ "-shaped POC1B, suggesting that their morphology correlates. $p=0.016$ by Chi-squared. $\mathbf{g}$ Model of the DC in the ejaculated spermatozoon based on electron microscopy, confocal microscopy, and 3D-SIM. Sc striated columns, Ax axoneme, $\mathrm{V}$ vault. Scale bars $1 \mu \mathrm{m}$

$\mathrm{PC}$ and DC were more intense than in the midpiece axoneme, possibly because the PC and DC microtubules were unmasked during centrosome reduction. These findings suggest that the DC microtubules were not degenerated as was previously believed.

To determine the location of the DC's microtubules, we used transmission electron microscopy (TEM) with high-pressure freezing-freeze substitution, which better preserves cellular structural integrity when compared to chemical fixation. We found that the DC microtubules splayed out from the axoneme, surrounding the vault, and extended up to the PC (Fig. 2c, d). The splayed microtubules extend from the cylindrical axoneme and open up forming an inverted cone that ends with an ovoid base facing the nucleus (Fig. 2e). The presence of these microtubules may have been overlooked previously because they were not in the typical nine-fold symmetric pattern (Fig. 2f) and they may have been disrupted by the classical chemical fixation methods used for TEM. Structurally atypical centrioles have been observed previously in insect sperm and are known as the proximal centriole-like 
a
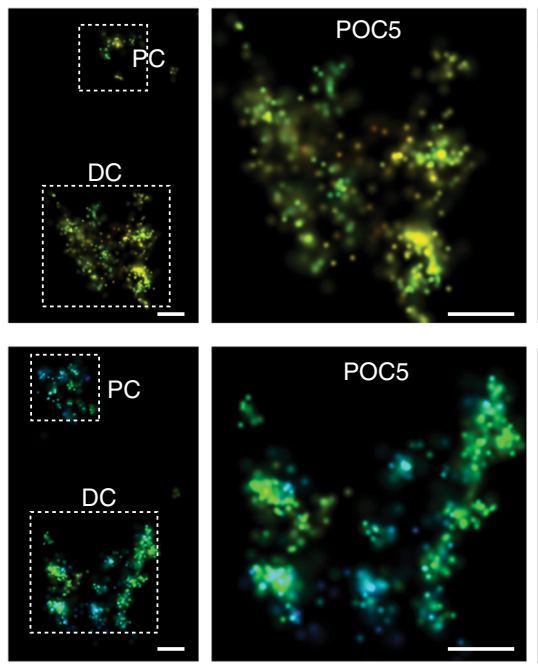

b

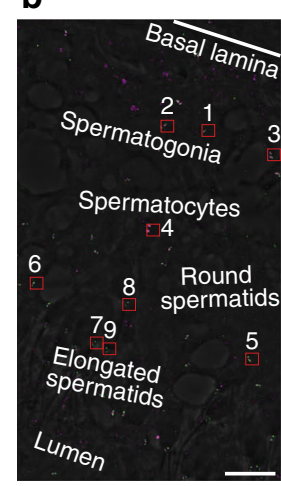

Spermatogonia

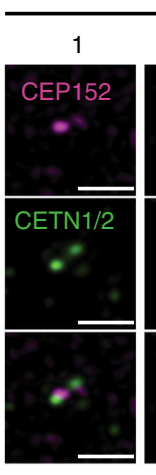

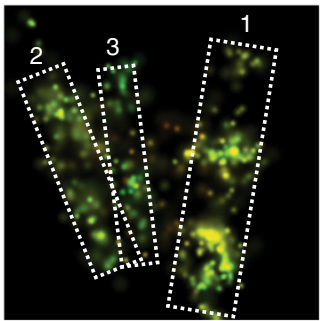

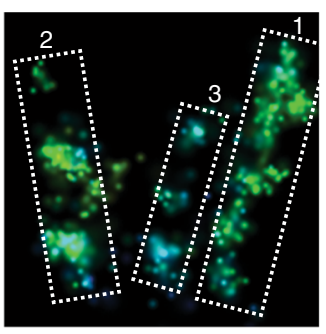

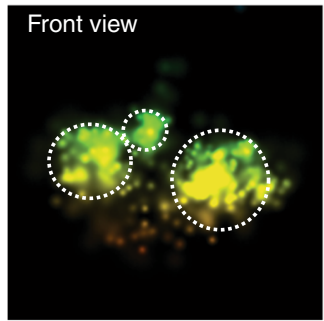

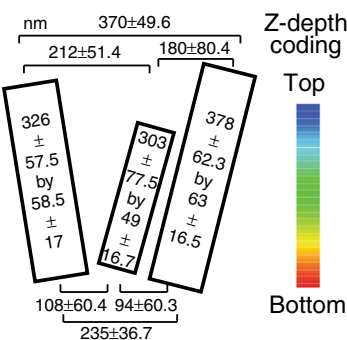

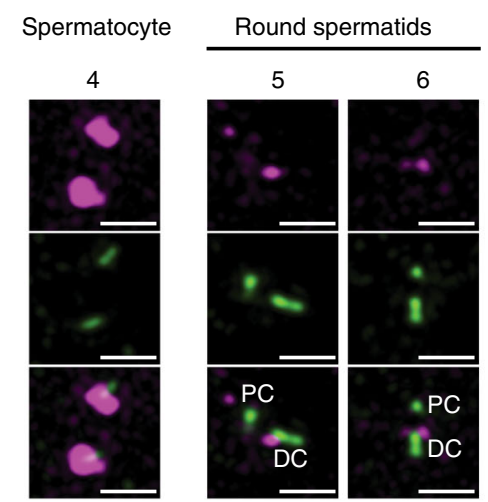
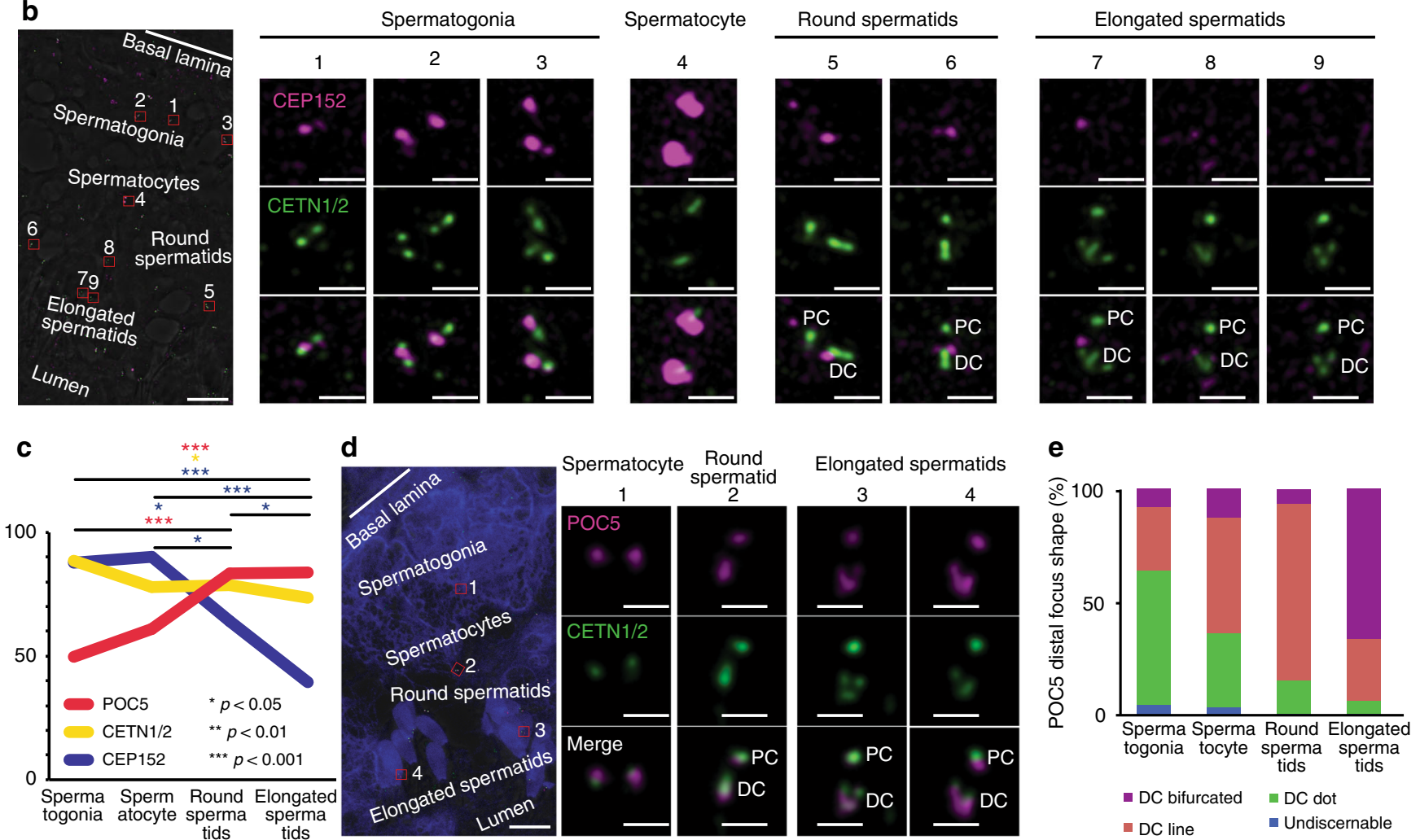

Fig. 4 POC5 and CETN $1 / 2$ are enriched and redistributed, while CEP152 is reduced. a STORM with POC5 antibodies recognized the PC and DC (left panel). Zoom in on the DC (two middle panels) identifies two major rods (marked as "1" and "2") and one minor rod (marked as " 3 "). A diagram (second row, right panel) depicting the dimensions of the rods and their distances from each other (from center to center) at their tips and bases $(N \geq 7)$. Scale bar $100 \mathrm{~nm}$. b A section of a seminiferous tubule (left panel) depicting the changes in CEP152 (pink) and CETN1/2 (green) in various stages (cells are numbered 1-9). During spermatogenesis, CEP152 and CENT1/2 localizes to 2-4 foci in spermatogonia (cells 1-3). In spermatocytes, CEP152 is maintained, often in large foci surrounding much smaller CENT1/2 lines (cell 4). In round spermatids, CEP152 is localized to the ends of elongated CENT1/2 foci and reduces into two small foci straddling the CENT1/2 line (cells 5-6). Finally, in the elongated spermatids (cells 7-9), CEP152 is localized to the tips of the CENT1/2 "V" shape, before it is finally dramatically reduced. Scale bar $10 \mu \mathrm{m}$ in low-magnification images (left), and $1 \mu \mathrm{m}$ in centriole high-magnification images (right). c During spermatogenesis, CEP152 is dramatically reduced (blue), CETN1/2 is slightly reduced (yellow), and POC5 is enriched (red) ( $\mathrm{Z} \geq$ 3). $p$-values determined by ANOVA with Fisher's LSD post hoc. $\mathbf{d}$ A section of a seminiferous tubule (left panel) depicting the changes in the distribution of POC5 (pink) and CETN1/2 (green) in various stages (cells 1-4). Both POC5 and CENT1/2 localize to dot-shaped centrioles in spermatocytes (cell 1). In round spermatids, both POC5 and CENT1/2 elongate into a DC that is longer than the PC (cell 2). Finally, in the elongated spermatids (cells 3-4) both CETN1/2 and POC5 appear as " $\mathrm{V}$ " shapes. Scale bar $10 \mu \mathrm{m}$ in low-magnification images (left), and $1 \mu \mathrm{m}$ in centriole high-magnification images (right). e Quantification of the DC shape by POC5 immunolabeling during spermatogenesis. Most spermatogonia are dot shaped; most round spermatids are elongated; and most elongated spermatids are bifurcated $(N \geq 3, p \leq 0.00001)$. $p$ by Chi-squared 

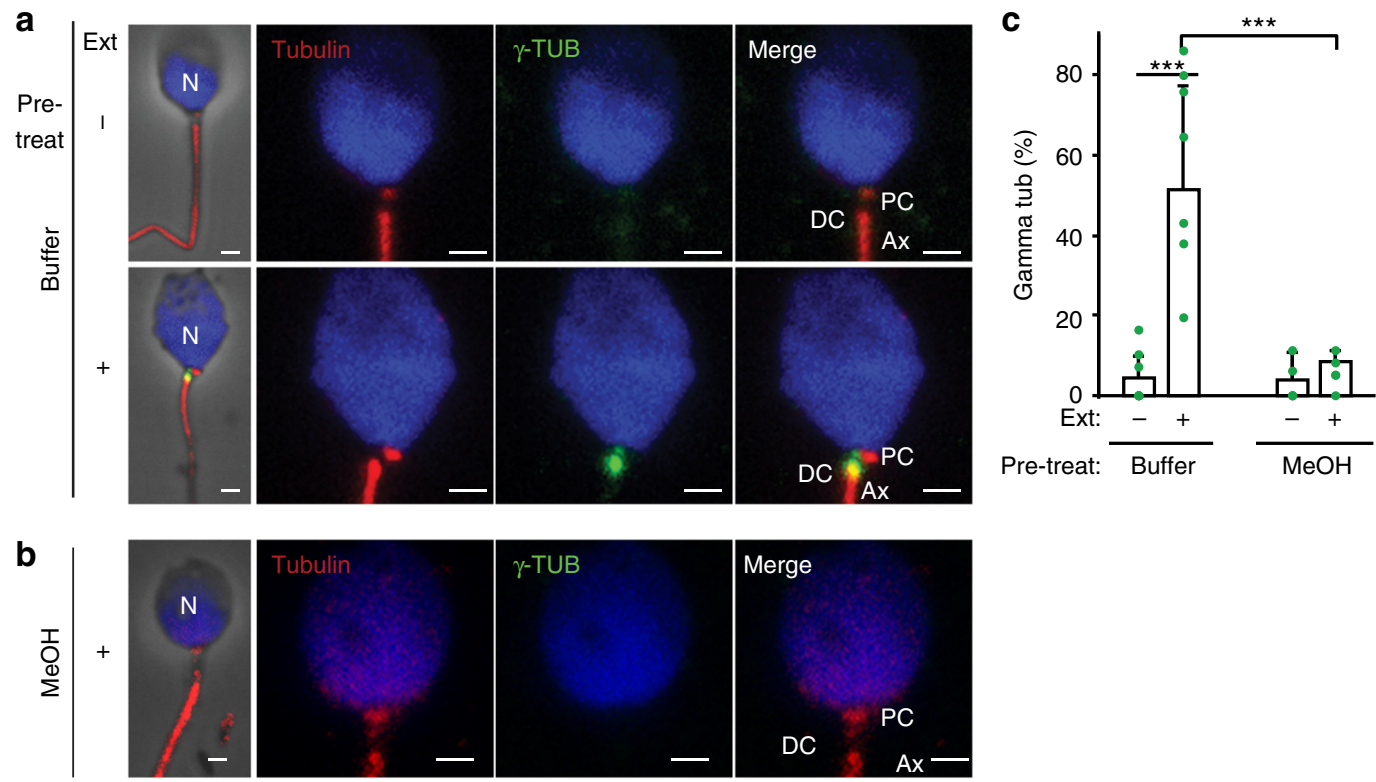

Fig. 5 The remodeled DC is functional in vitro. a-c The human remodeled DC recruits $\gamma$-tubulin from Xenopus egg extract. Demembranated human ejaculated spermatozoa did not have $\gamma$-tubulin, but after exposure to the extract, $\gamma$-tubulin was present near the DC (a). Demembranated spermatozoa

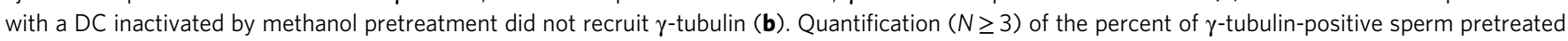
with either buffer or methanol $(\mathrm{MeOH})$ and then exposed to extract $(+)$ or buffer $(-)(\mathbf{c})$. $p<0.001$ by $t$-test. Scale bar $1 \mu$ m. Error bars represent +1 standard deviation

structures $(\mathrm{PCL})^{15,17,25-27}$. However, the atypical DC is distinct from that of the PCL in fly (Fig. $2 \mathrm{~g}$ ) or beetle (Fig. 2h), suggesting that sperm centrioles evolve diverse structures.

The DC proteins are organized into rods. To precisely localize the DC proteins, we performed sub-diffraction resolution microscopy. We found that CEP63, which normally forms a ring around the centriole, localized as two dots, and CPAP, which is commonly found in the centriole, localized around the DC (Supplementary Fig. 4a-b). Importantly, POC1B, CETN1/2, and POC5 exhibited rod-like distribution in various orientations (Supplementary Fig. 4c). We identified six types of orientations. The first, most common, was "V" shaped, where the rods were angled away from each other. The second was "Reduced" orientation, when one or both rods had a reduced size, appearing as a single rod, a rod and a dot, a dot, or no signal at all. Third was "Parallel", where the rods were parallel. The fourth orientation we found was "Wide", where presumably, the rods were close to each other. Fifth was "Staggered", where the rods were in various orientations. And finally, the sixth orientation was "Ring", where the rods appeared to be connected by thin lines (Fig. 3a). Four of these categories included rods in different orientations ("V", "Parallel", "Wide", and "Staggered"), but the two others cannot be explained as a different view of the same configuration, suggesting that the relative position between the rods may be varied. However, in $40 \%$ of spermatozoa imaged, the rods appeared in a " $V$ " shape suggesting this may be the preferred orientation (Fig. 3b, c). A similar "V" shape was also observed in STED (Supplementary Fig. 4d).

The length of the individual rods in the "V" is about $190 \mathrm{~nm}$, which is about half the length of the distal lumen of a typical centriole $(\sim 350 \mathrm{~nm})^{28,29}$ (Fig. 3d). The width of the "V" tip opening is about $230 \mathrm{~nm}$, which is much wider than a typical centriole lumen $(\sim 130 \mathrm{~nm})^{28}$. The "V" was in line with the axoneme and was flanked by the splayed-out DC microtubules (Fig. 3e, f). This suggests that the rods associate with the splayed microtubules of the DC.
Further examination of our electron microscopy images finds electron-dense material along the splaying DC microtubules that might be the structure underlying the rods (Supplementary Fig. 5). Similarly, Manandhar et al. ${ }^{5}$ found using immunogold labeling that CETN1/2 associates with microtubules at the point where the microtubules begin to splay in the DC, the same site where we observed the DC rods. This rod organization has never been described for any centriolar protein; most centriolar proteins and the structures within the distal lumen of a centriole exhibit radial symmetry in the form of disk and column structures ${ }^{30-33}$ Together, these findings demonstrate that several centrosomal proteins have an atypical distribution in the DC (Fig. $3 \mathrm{~g}$ ).

Bovine DC undergo remodeling. To further study centrosome remodeling and DC function during sperm development, we examined bovine testes because bovine sperm, like human sperm, was thought to contain only the $\mathrm{PC}^{12,34}$. Like human spermatozoa, POC1B, POC5, CETN1/2, and tubulin labeled the DC of bovine spermatozoa (Supplementary Fig. 6a-b), and the spermatozoa lack CEP152 (an essential PCM protein) and SAS-6 (a daughter centriole marker) (Supplementary Fig. 6b-c). Furthermore, the CEP152, SAS-6, POC1B, POC5, and CETN1/2 antibodies recognize the centrosomes of undifferentiated bovine cells, validating the antibody specificity (Supplementary Fig. 6d-h). In addition, bovine spermatozoa shows an obvious "V" shape in the DC using HyVolution confocal microscopy, suggesting that the bovine DC is both similar in shape to, and bigger than the human DC (Supplementary Fig. 6i-j). Together, the compositional similarities and size increase make bovine sperm a good model to examine the finer detail of the DC and its development.

The advantages of bovine sperm allowed us to closer examination of the DC rods using stochastic optical reconstruction microscopy (STORM), which has a resolution limit of $\sim 30$ $\mathrm{nm}$. In a side-view projection, we found that the bovine DC is made of two main rods and one minor rod (Fig. 4a). Likewise, a $90^{\circ}$ rotation projection clearly showed three dots representing a top-view of the rods. The two major rods are about $350 \mathrm{~nm}$ long, 
a

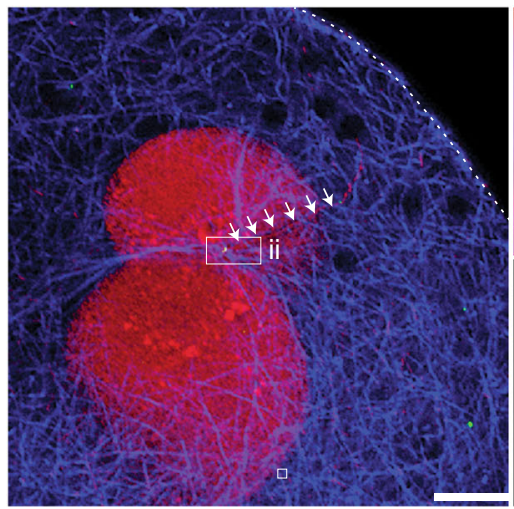

b

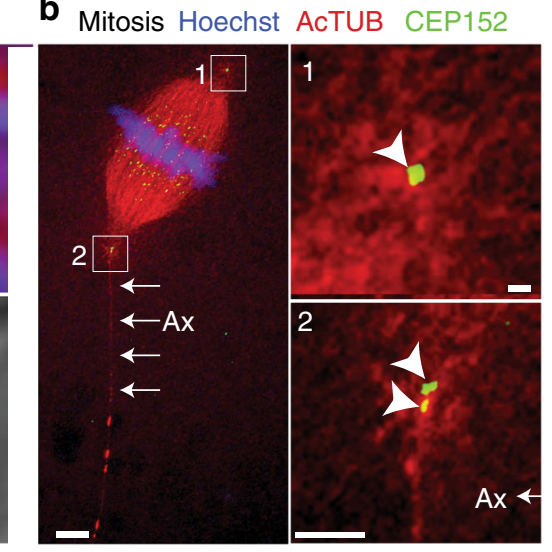

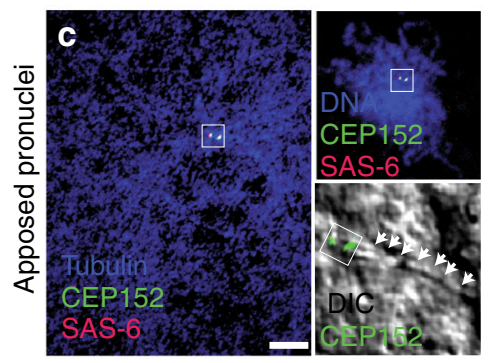
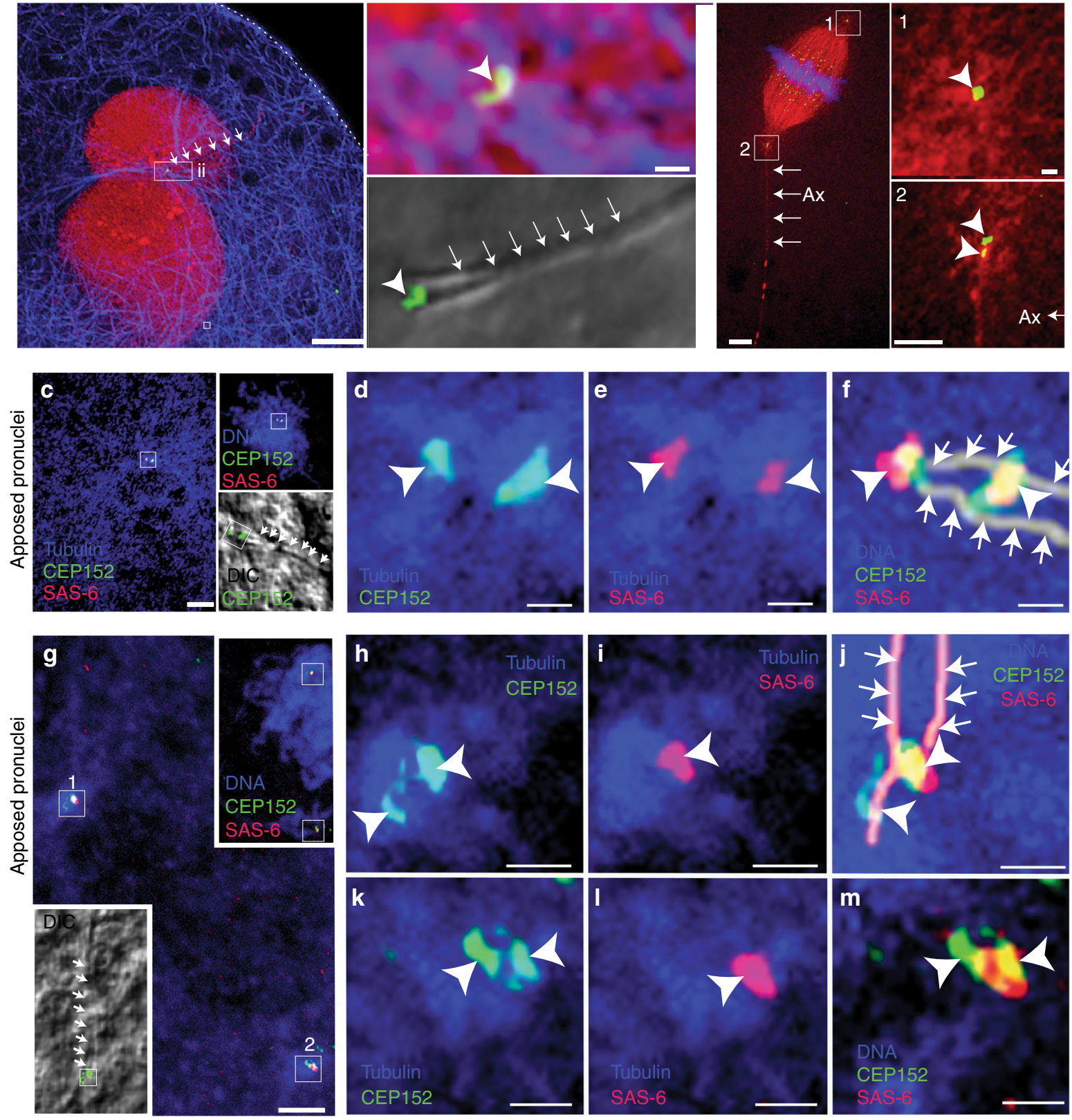

Fig. 6 The remodeled DC is functional in bovine zygotes. a, b The axoneme with a CEP152-labeled DC was observed near the male pronucleus (a) and at the spindle poles (b). a Adjacent pronuclei (red), and the axoneme (arrows) at low magnification on the left. A pair of CEP152-labeled centrioles (arrowhead, right side high magnification) are found at the axoneme base. The bifurcated axoneme (arrows) is observable using DIC (lower right high magnification). b A spindle (red) with an attached axoneme (red, Ax, arrows) and a pair of CEP152-labeled centrioles (green, arrowheads, pole 2) at one pole and CEP152-labeled centrioles at the other pole (green, arrowhead, pole 1). Centrioles are within an aster (red). Scale bar $10 \mu \mathrm{m}$ (left), and $1 \mu \mathrm{m}$ (right). c-f Zygote with closely apposed pronuclei with centrioles retained at axoneme base has separated centrioles co-labeled with CEP152 (green) and SAS-6 (red) within a single aster (blue) (f). The upper inset in c shows the centrioles relative to the pronuclei (blue). The lower inset in c shows a DIC image of the axoneme (arrows) overlaid with CEP152-labeled centrioles (box, green). The CEP152 (green, arrowheads, d) and SAS-6-labeled centrioles are within an aster (blue, arrowheads, e). The composite image (f) shows the centrioles (arrowheads) at the bifurcated axoneme base (arrows). Scale bar 10 $\mu \mathrm{m}$ (left), and $1 \mu \mathrm{m}$ (right). g-m A zygote with two separated centriole pairs (box " 1 " and " 2 " in $\mathbf{g}$ that each reside in an aster; blue). Upper inset in $\mathbf{g}$, shows DNA (blue), and the labeled centrioles (boxes) relative to the pronucleus (blue). Lower inset in $\mathbf{g}$ shows a DIC image of the bifurcated axoneme (arrows) and CEP152-labeled centriole pair 1 (box). h-j Enlarged images of centriole pair 1 showing details of CEP152 (green, arrowheads, h) and SAS-6 (red, arrowheads, $\mathbf{j}$ ) within the aster (blue). Image $\mathbf{j}$ shows centriole pair 1 (arrowheads) at the bifurcated axoneme base (arrows). $\mathbf{k}-\mathbf{m}$ Enlarged images of centriole pair 2 showing CEP152 (green, arrowhead, k) and SAS-6 (red, arrowheads, I) within the aster (blue). Image (m) shows centriole pair 2 (arrowheads). Scale bar $10 \mu \mathrm{m}$ (left), and $1 \mu \mathrm{m}$ (right) 
whereas the minor rod is only about $300 \mathrm{~nm}$ long. The length is twice as long as the human DC rods, which agrees with our findings using HyVolution confocal microscopy (Figs. 3d, 4a, Supplementary Fig. 6j). The distance between the tops of the rods is approximately $370 \mathrm{~nm}$, which is substantially wider than a typical centriole. The distance between the rods at the base is only $230 \mathrm{~nm}$, which is similar to the width of a typical centriole lumen. Together, these dimensions suggest that the base of the rod's "V" shape is around the same size as a typical centriole lumen, but then opens up much wider than a typical centriole.

To gain insight into the process of DC remodeling, we tracked centrosomal proteins during bovine spermatogenesis. We found that while CEP152 is present in spermatogonia, spermatocytes, and round spermatids, its levels are dramatically reduced in elongated spermatids (Fig. 4b, c). In contrast, we observed that as CEP152 is dramatically reduced (by $\sim 55 \%$ ), CETN1/2 is slightly reduced (by $\sim 17 \%$ ), and POC5 is enriched (by $\sim 68 \%$ ) (Fig. 4c, d). A close look at POC5 localization revealed that POC5 and CETN1/2 transforms from a rounded focus, to an elongated focus, and then to " $\mathrm{V}$ " during the transition from round spermatids to elongated spermatids (Fig. 4b, d, e). These findings suggest that centriole remodeling is accompanied by both protein reduction and protein enrichment and that the structure of the DC is remodeled during spermiogenesis, before the sperm has gained mobility.

The human DC is competent in vitro. To examine the function of the DC, we used a cell-free centrosome reconstitution system that exposes demembranated sperm to Xenopus egg extracts ${ }^{34,35}$. The exposed sperm centrioles can recruit PCM proteins typically involved in aster formation from the egg extract, thus simulating the fertilization environment without creating embryos. This system has previously demonstrated the essential role of the PCM protein $\gamma$-tubulin in centrosome assembly and function ${ }^{35}$. Later, using human sperm, it was found that the sperm centrosome was able to recruit $\gamma$-tubulin, but the specific site of recruitment was not investigated $^{34}$. We employed this system to determine if the DC was capable of recruiting $\gamma$-tubulin and found that the main site of $\gamma$ tubulin recruitment was near the DC (Fig. 5a). This $\gamma$-tubulin recruitment activity was specific to the sperm, as sperm fixed with methanol were unable to recruit $\gamma$-tubulin (Fig. 5b, c). Altogether, this suggests that the remodeled DC is competent to recruit PCM.

The bovine DC functions in zygote. Since the DC in human spermatozoa is competent to recruit PCM, it may function as the second centriole of the zygote. Indeed, in human zygotes, the sperm axoneme is incorporated into the zygote where its base associates with an aster near the male pronucleus that is known as the sperm aster. Later during mitosis, the incorporated sperm axoneme associates with one spindle pole ${ }^{34,36}$. This association with the aster and the spindle pole could be an indication that the axoneme-attached DC is functional.

To test this, we examined the ability of the DC in bovine zygotes to recruit the PCM protein CEP152. In zygotes with adjacent male and female pronuclei, we found a pair of CEP152labeled centrioles at the axoneme base, abutting a pronucleus. This suggests that the DC recruits PCM and forms a functional centrosome (Fig. 6a and Supplementary Fig. 7a-d). Later, in mitotic metaphase, we observed CEP152-labeled centrioles at both poles of the bovine spindle. At the pole with the attached sperm axoneme, we found a pair of CEP152-labeled centrioles (Fig. 6b and Supplementary Fig. 7c). This suggests that the DC also participates in spindle pole formation. Together, these findings suggest that the DC functions as a centriole in the zygote.

Similar to prior work, we observed by differential interference contrast (DIC) optics that the axoneme base nearest the paternal pronucleus often splays into two fibers ${ }^{34,37}$ (Fig. 6a). This axoneme base associates with the center of a microtubule aster, which splits later to generate a second aster (Supplementary Fig. 7d).

To determine if the DC was able to function as a platform for the formation of a new daughter centriole, we labeled zygotes with the daughter centriole protein SAS- 6 . We found that in zygotes with closely apposed male and female pronuclei the centrioles were retained at the bifurcated axoneme base (Fig. $6 \mathrm{c}$ and Supplementary Fig. $7 \mathrm{~d}$ ). These centrioles were co-labeled with CEP152 and SAS-6 within a single microtubule aster (Fig. 6c-f and Supplementary Fig. 7d). Later, when the sperm aster splits into two, we found a pair of centrioles at the center of each microtubule aster. Each of these pairs contains two CEP152 foci, only one of which is associated with a SAS-6 focus (Fig. $6 \mathrm{~g}-\mathrm{m}$ ). One of the pairs is associated with the base of the bifurcated sperm axoneme (Fig. 6g-j and Supplementary Fig. 7d). Together, this suggests that centriole duplication occurs before aster duplication, and when it takes place a single daughter centriole forms in association with each of the sperm centrioles. Importantly, this means that the atypical DC is able to act as platform for the formation of a daughter centriole.

\section{Discussion}

In summary, we have found that the mammalian sperm centrosome is remodeled during spermiogenesis. During the remodeling, centrosomal proteins were eliminated, reduced, or enriched. Many of these proteins were redistributed between the DC, PC, and PCM. The centriole distal lumen proteins were redistributed in the DC to form rods that associated with the splayed DC microtubules. As a result, the remodeled DC had a general appearance of truncated ovoid cone. The sperm provided the zygote with two centrioles, a typical centriole (the PC) and an atypical centriole attached to the axoneme base (the remodeled DC), as well as a remodeled, structural PCM, the striated columns and the capitulum. The DC was atypical but functional; in vitro, it recruited $\gamma$-tubulin. In vivo, the DC recruited PCM, formed a daughter centriole, and localized to one spindle pole in the zygote. Altogether, our findings support a paternal inheritance model where the sperm provides two functional centrioles, of varied structure and composition, along with the associated PCM/striated columns structures.

While our findings elucidate the origin of the zygote's centrioles, they reveal a new enigma. The presence of a functional, yet atypical DC raises the question of the purpose of the remodeled structure. We have found previously that the Drosophila atypical centriole is essential for normal fertility and embryo development ${ }^{15}$, suggesting that the human atypical DC may be instrumental in fertility. The difference between the typical and atypical centrioles may provide a cue for early embryonic development ${ }^{38}$. Furthermore, it is possible that the remodeling step allows for some flexibility in the neck region for sperm movement.

Additionally, our work raises questions regarding the fate of the DC. If the DC remains attached to the bifurcated axoneme, as we have observed, it does not appear capable of forming a cilium. This may not be a problem as primary cilia form only in some cell fates (the epiblast) at much later stages (post-implantation) ${ }^{39}$. This work also has implications beyond the scope of reproductive biology; because several other differentiated cell types are thought to lack centrioles, it is possible that they have atypical centrioles ${ }^{40,41}$.

Mammalian and insect sperm were thought to carry a single functional centriole to the zygote. However, recently a second centriole that is atypical was described in insect sperm $^{15,16,25,42}$ Similarly, here we show that mammalian sperm also have one 
typical and one atypical centriole. Together, these observations argue for an evolutionarily conserved sperm centriole number with variable structure, microtubule organization, and protein composition. This conservation suggests that sperm centrioles and their remodeling could play a critical role in fertility and early embryo development. Understanding the precise mechanisms of centrosome transmission during reproduction may help solve currently idiopathic forms of male infertility, generate novel targets for male contraception ${ }^{43}$, and even support organelle donation strategies to treat centriole-mediated infertility ${ }^{44}$.

\section{Methods}

Sperm preparation. Ejaculated spermatozoa were obtained from Coba Select Sires (bovine) and Manhattan Cryobank and Fairfax Cryobank (human). In all animals tested, motile ejaculated spermatozoa were washed and selected using a PureCeption density gradient according to instructions (Origio, ART-2004). All human sperm samples were acquired after approval from the University of Toledo's Institutional Review Board and this work was declared exempt.

U2OS cells. U2OS cell were obtained from Dr. Deborah Chadee (University of Toledo). They were grown in Dulbecco's Modified Eagle's Medium (DMEM; Mediatech), supplemented with $10 \%$ fetal calf serum (FCS; Atlanta Biologicals), at $37^{\circ} \mathrm{C}$ in a humidified atmosphere supplemented with $5 \% \mathrm{CO}_{2}$.

Bovine cells and testes. TE11 p39 Bovine embryonic lung fibroblasts were obtained from Dr. Toshihiko Ezashi and Dr. R. Michael Roberts of the University of Missouri-Columbia. Bovine embryonic lung fibroblasts from 99-day fetuses were generated by Dr. Neil Talbot in 2009. They were grown in DMEM (Mediatech), supplemented with $10 \%$ FCS (Atlanta Biologicals), at $37^{\circ} \mathrm{C}$ in a humidified atmosphere supplemented with $5 \% \mathrm{CO}_{2}$. Bovine testes were purchased from Scholl's slaughterhouse in Blissfield Michigan. They were dissected and embedded fresh in OCT (EMS Diasum 62550-01). The OCT-embedded testes were frozen on dry ice and then sectioned. The $10 \mu \mathrm{m}$ sections were stained in the immunofluorescence protocol described below. As this work uses discarded tissue, no approval was required from University of Toledo's Institutional Animal Care and Use Committee.

Plasmids. POC1B312-406-His (amino acids 312-406 of NP_758440.1) in pQE10 vector were obtained from Dr. Chad Pearson ${ }^{45}$. POC1B312-406-GST was generated using the QuickFusion kit to move a POC1B312-406 fragment from the pQE10 vector into a pGEX-3× plasmid between restriction enzymes Xmal and EcoR1.

Protein purification. POC1B312-406 was grown in E. coli at $25^{\circ} \mathrm{C}$ in lysogeny broth with $100 \mu \mathrm{g} / \mathrm{mL}$ ampicillin and then stored at $4{ }^{\circ} \mathrm{C}$ for $3 \mathrm{~h}$ to overnight; we then added $1 \mathrm{mM}$ IPTG and incubated at $20^{\circ} \mathrm{C}$ for $3 \mathrm{~h}$. The bacteria were then centrifuged for $20 \mathrm{~min}$ at $12,000 \times \mathrm{g}$, and the supernatant was discarded. The pellet was resuspended in lysis buffer ( $50 \mathrm{mM}$ TRIS, $105 \mathrm{mM} \mathrm{NaCl}, 5 \%$ glycerol, and complete protease inhibitor cocktail (Roche)) and sonicated for $1 \mathrm{~min}$ at $10 \mathrm{~s}$ intervals at $40 \%$ power. The sonicated cells were then centrifuged at $20,000 \times g$ for $30 \mathrm{~min}$. POC1B312-406-His was purified by nickel beads, and POC1B312-406GST was purified by glutathione bead affinity column. Purified protein was separated on an SDS-Page gel, and corresponding bands were excised and used to immunize rabbit and rats.

Antibodies. Antibodies were generated against purified POC1B312-406-His. Polyclonal antibodies 537 and 538 were generated in rabbits by Pacific Immunology. Monoclonal antibodies (5G5A7 and 5G5A10) were generated in rat by ProMab using the purified POC1B312-406-His. Antibodies 537 and 538 were affinity purified by Pacific Immunology using purified POC1B312-406-GST. Antibodies 537 and 538 were used at a 1:100 dilution for immunofluorescence. The rat monoclonals were used at 1:20 for immunofluorescence. The polyclonal antibodies and rat monoclonal did not work well for western. All other antibodies used are described in Supplementary Table 1.

Immunofluorescence. For immunofluorescence, $7 \mu \mathrm{L}$ of PureCeption cleaned spermatozoa were placed on a poly-lysine slide (Sigma-Aldrich, P5899) and a Sigmacote coverslip (Sigma, SL2) was placed on top. The whole slide was then snap frozen and stored in liquid nitrogen. Sperm slides were stored in liquid nitrogen; when withdrawn, the coverslip was removed using forceps, and the slide was placed in a pre-chilled Coplin jar of ice-cold methanol for $2 \mathrm{~min}$. Next, the slide was placed in $1 \times$ phosphate buffered saline (PBS) for $1 \mathrm{~min}$, then placed for $60 \mathrm{~min}$ in fresh $1 \times$ PBS with $3 \%$ Triton X-1000 at room temperature. PBST-B was prepared by adding $1 \%$ bovine serum albumin (BSA) to PBST, and slides were then placed in PBST-B for $30 \mathrm{~min}$. Primary antibodies diluted in PBST-B were added to slides at the concentrations listed in Supplementary Table 1, after which the slides were covered in parafilm, placed in a humidity chamber, and incubated at room temperature for $1 \mathrm{~h}$ or overnight at $4{ }^{\circ} \mathrm{C}$. The slides were washed three times in PBST for $5 \mathrm{~min}$ each Next, the secondary antibody mixture was prepared by combining PBST, secondary antibodies (see Supplementary Table 1B), and Hoechst 33258. The secondary antibody mixture was added to slides, and they were covered in parafilm and incubated for $>1 \mathrm{~h}$ at room temperature. Slides were then washed three times with PBST for 5 min each, followed by three times with $1 \times$ PBS for 5 min each. Finally, the slides were sealed and imaged using a Leica Sp8 confocal microscope and some images (Fig. 4) were processed using a Leica HyVolution 2 System.

Sperm images were taken at a magnification of $640 \times$ and zoom of $6 \times$, with $512 \times 512$ pixel density. Using Photoshop, immunofluorescence sperm images were cropped to 200 pixels by 100 pixels, or 100 pixels by 100 pixels.

Immunofluorescence U2OS images were cropped to 150 pixels by 150 pixels or 25 pixels by 25 pixels. The intensity was modified to allow easy visualization, and the panels were resized to 300 DPI for publication. All photon counting was done with a constant laser power that, in various experiments, ranged between 0.25 and $2 \%$, using CETN1/2, tubulin, or POC1B as a reference for centriole location.

Bovine zygote images in Fig. 4d were taken using a Nikon Al four laser line confocal microscope equipped with elements acquisition and analyses software and were deconvolved. Multiple pictures of the bovine zygotes were taken, resulting in some bleaching in areas that were taken at higher zoom.

Electron microscopy with high-pressure freezing. For TEM analysis of human sperm centrioles, the ejaculated sperm were centrifuged in Eppendorf tubes (1.5 $\mathrm{mL}$ ) at $1000 \mathrm{rpm}$ for $1 \mathrm{~min}$, and then the pellets were processed using the highpressure freezer system (Leica EM HPM100) in 20\% BSA. The frozen samples were dehydrated and stained en bloc using a freeze substitution preprocessor (Leica EM AFS2) in $96 \%$ acetone with $1.5 \% \mathrm{OsO}_{4}$ (osmium crystals dissolved in acetone and $4 \%$ water). The freeze substitution started with $-90^{\circ} \mathrm{C}$ for $6 \mathrm{~h}$, then warmed from -90 to $-10^{\circ} \mathrm{C}$ over $15 \mathrm{~h}\left(5.3^{\circ}\right.$ slope). Then the samples were warmed to $-3^{\circ} \mathrm{C}$ over $1 \mathrm{~h}\left(7^{\circ}\right.$ slope) while being washed with $96 \%$ acetone (at $\left.-7^{\circ} \mathrm{C}\right)$. They were then warmed from -3 to $4^{\circ} \mathrm{C}$ over $1 \mathrm{~h}\left(7^{\circ}\right.$ slope) while being washed twice with $100 \%$ acetone. Lastly, the dehydrated samples were infiltrated and embedded in EMbed 812 resin while warming to room temperature. Ultrathin sectioning (70 $\mathrm{nm}$ ) was performed using an ultramicrotome (Leica EM UC6), and sections were post-stained with $6 \%$ uranyl acetate (in 1:1 70\% ethanol and $100 \%$ methanol), and Reynolds' lead citrate (3-4\% in preboiled double-distilled $\mathrm{H}_{2} \mathrm{O}$ ). The sections were imaged using TEM (JEOL 1400-plus), operating at $80 \mathrm{kV}$.

Correlative light and electron microscopy. Ejaculated sperm was obtained from Fairfax CryoBank, and cleaned using the PureCeption kit (Origio, ART-2004). Sperm cells were then immobilized on Poly L-lysine-coated coverslips, fixed with $1.5 \%$ glutaraldehyde in PBS, permeabilized with $0.3 \%$ Triton-X-100, and immunolabeled with POC1B antibody (as described above). The coverslip was mounted in the imaging chamber, 200-nm-thick Z-sections through the sperm cells were recorded to mark the position of fluorescent signals within the cells using a Nikon Eclipse Ti inverted microscope equipped with a $13-\mu \mathrm{m}$ pixel DU888 camera (Andor) using a $100 \times$ NA 1.42 Plan Apo objective. High-magnification and lowmagnification $(100 \times$ and $20 \times$ ) DIC images were additionally recorded to ascertain the position of sperm cells. Samples were then prepared for electron microscopy analysis according to the standard protocol. Briefly, samples were pre-stained with $2 \%$ osmium tetroxide and $1 \%$ uranyl acetate, dehydrated, and embedded in EMbed 812 resin. 80 -nm-thick serial sections were made, post-stained with uranyl acetate and lead citrate, and imaged using a transmission electron microscope (Hitachi) operating at $80 \mathrm{kV}$. Image analysis and the alignment of the serial sections were performed using Fiji and Photoshop.

Super resolution microscopy. 3D-SIM data were acquired using an Elyra PS.1 microscope from Carl Zeiss, equipped with a plan-apochromat $63 \times / 1.4$ oilimmersion objective lens with an additional $1.6 \times$ optovar. Images were collected with an Andor iXon 885 EMCCD camera, resulting in a raw data pixel size of 79 $\mathrm{nm}$. Z-stacks were acquired with a spacing of $101 \mathrm{~nm} /$ pixel. The fluorophores were excited with a $200-\mathrm{mW} 488-\mathrm{nm}$ laser. Images were acquired with a laser power, at the objective focal plane, of $52.6 \mathrm{~mW}$, attenuated to $5 \%$. Exposure times were between 50-200 ms and EMCCD camera gain values between 5-20. Five phases at each of three rotation angles $\left(-75^{\circ},-15^{\circ},+45^{\circ}\right)$ of the grid excitation pattern were acquired. A 495-550 band-pass filter was used to collect fluorescence from Alexa 488 antibody-labeled samples. A 570-620 band-pass filter was used to collect fluorescence excited with a wavelength of $555 \mathrm{~nm} .5-8 \%$ power intensity was used. The data were processed using the SIM module of the Zen software, version 8.1, with Weiner filter between $10-3$ and 10-5. Super resolution sperm images were cropped to $50 \times 50$ pixels.

Bovine sperm was attached to $25 \mathrm{~mm}, 1.5$, high precision cover glasses (Warner Instruments), as described above for immunofluorescence. Anti-POC5 antibody (Thermo Fisher PA5-24308) was used at 1:70. CF647-conjugated FAB2 antibodies (Biotium) were used at 1:800 dilution to label primary antibodies. The anti-POC5 primary antibody was labeled by a Goat anti-rabbit Alexa 647 secondary antibody (Biotum) at 1:800 dilution. Before STORM imaging, samples were layered with 100 $\mathrm{nm}$ tetra-spectral fluorescent spheres (Invitrogen), which served as fiducial 
markers. Coverslips were mounted to Attofluor Cell chambers (Thermo Fisher) in imaging buffer ( $10 \%$ dextrose in $100 \mathrm{mM}$ Tris at $\mathrm{pH} 8.0,25 \mathrm{mM} \beta$ mercaptoethylamine, $0.5 \mathrm{mg} / \mathrm{mL}$ glucose oxidase, and $67 \mu \mathrm{g} / \mathrm{mL}$ catalase). 3D STORM imaging was performed on a Nikon N-STORM4.0 system using Eclipse Ti inverted microscope, Apo TIRF 100× SA NA 1.49 Plan Apo oil objective, 405, 561, 488 , and $647 \mathrm{~nm}$ excitation laser launch (Agilent) and a back-illuminated EMCCD camera (Andor, DU897). The $647 \mathrm{~nm}$ laser line was used to promote fluorophore blinking. $405 \mathrm{~nm}$ laser was used to reactivate fluorophores. $561 \mathrm{~nm}$ laser was used to record the signals of fiducial markers. $\sim 20,000$ time points were acquired at a 50 $\mathrm{Hz}$ frame rate each 16-20 ms. NIS Elements (Nikon) was used to analyze and present the data.

STED images of bovine and human sperm were stained as described above and were acquired on a Leica SP8 with STED head at the University of Michigan.

Xenopus egg extract. Studies with Xenopus laevis were conducted in compliance with the U.S. Department of Health and Human Services' Guide for the Care and Use of Laboratory Animals and were approved by the University of Michigan Institutional Animal Care and Use Committee. Xenopus egg extract was generated according to the methods of Murray and Kirschner ${ }^{46}$, with modifications from Simerly et al. ${ }^{34}$ and Hannak and Heald ${ }^{47}$. In brief, Xenopus laevis females (obtained from Nasco) were induced to ovulate by injecting human chorionic gonadotropin (MP Biomedicals) into the dorsal lymph sac, and frogs were allowed to drop their eggs overnight in $\mathrm{Ca}^{2+}$-free $1 \times$ Marc's Modified Ringer's solution (MMR: $100 \mathrm{mM}$ $\mathrm{NaCl}, 2 \mathrm{mM} \mathrm{KCl}, 1 \mathrm{mM} \mathrm{MgCl}, 0.1 \mathrm{mM}$ ethylene glycol tetraacetic acid (EGTA), 5 mM HEPES, pH 7.8). Eggs were collected and dejellied using $2 \%$ cysteine in $\mathrm{Ca}^{2}$ ${ }^{+}$-free $1 \times$ MMR, pH 7.9, followed by washes in $50 \mathrm{~mL}$ each of extraction buffer (XB: $100 \mathrm{mM} \mathrm{KCl}, 1 \mathrm{mM} \mathrm{MgCl}, 10 \mathrm{mM}$ Hepes, $50 \mathrm{mM}$ Sucrose, $\mathrm{pH}$ 7.7), XBcytostatic factor (CSF) $\left(200 \mathrm{~mL} \mathrm{XB}, 200 \mu \mathrm{L} 1 \mathrm{M} \mathrm{MgCl}_{2}, 1 \mathrm{~mL} 1 \mathrm{M} \mathrm{K}\right.$-EGTA), and XB-CSF with protease inhibitors (XB-CSF with $10 \mu \mathrm{g} / \mathrm{mL}$ each of leupeptin, chymostatin, and pepstatin). Eggs were then transferred to a 4-mL Beckman UltraClear centrifuge tube (Beckman 344062), using a disposable plastic pipette with the tip cut off, taking care to make sure the eggs were not exposed to air. The 4-mL tube was placed inside a 13.2-mL Beckman Ultra-Clear centrifuge tube (Beckman $344059)$ as an adapter. The 4-mL tube was filled to the top with excess $\mathrm{XB}$, and $\mathrm{XB}$ was added to the outside of the $4-\mathrm{mL}$ tube so that the level of the $\mathrm{XB}$ outside the smaller tube matched the level of the eggs; this was done to prevent collapse of the tube during centrifugation. The tubes were centrifuged for $2 \mathrm{~min}$ at $1600 \mathrm{rpm}$ at 16 ${ }^{\circ} \mathrm{C}$ in a clinical centrifuge to pack the eggs. Excess liquid was removed using a transfer pipette fitted with a $200-\mu \mathrm{L}$ pipette tip. The tubes were then centrifuged at $11,500 \mathrm{rpm}$ for $15 \mathrm{~min}$ at $16^{\circ} \mathrm{C}$ using an SW41 hanging bucket rotor in a WX80 Sorvall ultracentrifuge to crush the eggs. The side of the tubes was pierced using an 18 -gauge needle, and the straw-colored cytoplasmic layer was drawn into a 1-mL syringe, taking care to avoid the vesicles close to the lipids at the top of the cytoplasmic layer as well as the black nuclear layer below the cytoplasmic layer. The needle was removed from the syringe, and the cytoplasmic extract was transferred to an Eppendorf tube. The volume of the extract was estimated, then supplemented with 1:50 energy mix (190 mM creatine phosphate, $25 \mathrm{mM}$ ATP, $25 \mathrm{mM} \mathrm{MgCl}$, $2.5 \mathrm{mM}$ EGTA, pH 7.7), 1:1000 leupeptin, chymostatin, and pepstatin $(10 \mathrm{mg} / \mathrm{mL}$ stock), 1:3000 nocodazole (6 mg/mL stock), and 1:500 cytochalasin B $(5 \mathrm{mg} / \mathrm{mL}$ stock). This supplemented extract was stored for up to $2 \mathrm{~h}$ on ice prior to use. A "control buffer" was made by supplementing XB-CSF with the energy mix, protease inhibitors, nocodazole, and cytochalasin B.

PCM recruitment in vitro. PCM recruitment was preformed according to the methods of Murray and Kirschner ${ }^{46}$ with modifications from Simerly et $\mathrm{al}^{34}$. In brief, $50-100 \mu \mathrm{L}$ PureCeption-washed sperm (see sperm preparation) was placed onto a cleaned uncoated 12-mm circle glass coverslip (Fisher 12-545-81) and allowed to bind for 10-20 min. The unbound sperm was removed, and the bound sperm was permeabilized by adding $50-100 \mu \mathrm{L}$ of $0.1 \%$ lysophosphatidyl choline (Sigma L4129-25MG) diluted in Kenney's Modified Tyrode's medium (KMT: 100

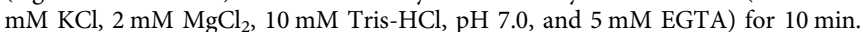
50-100 $\mu \mathrm{L}$ of Xenopus cell-free extract or control buffer was added to the coverslip for $45-60 \mathrm{~min}$ at $37^{\circ} \mathrm{C}$. Coverslips were washed in KMT before being fixed in $20^{\circ} \mathrm{C}$ cold methanol $(5 \mathrm{~min})$ and immunostained as described above. For the methanol pretreatments, the coverslips were fixed for $5 \mathrm{~min}$ in ice-cold methanol or 3.7\% formaldehyde at room temperature after permeabilization and before the extract was added. Then they were washed in KMT before resuming the PCM recruitment protocol.

Zygotes. Bovine in vitro fertilization and staining were performed as described previously ${ }^{36,48}$. In brief, bovine oocytes were acquired from a slaughterhouse and fertilized by Applied Reproductive Technology (AppliedReproTech). As this work uses commercially available cells, approval was not required from University of Toledo's Institutional Animal Care and Use Committee. They were shipped in fertilization media (supplied by AppliedReproTech) overnight in an incubator at $39^{\circ} \mathrm{C}$. Upon receipt, cumulus cells were removed using a stripper pipette with $135-$ $\mu \mathrm{m}$ tips (Origio MXL3-135) in the D1 growth media (provided by AppliedReproTech). Then cumulus-free zygotes were cultured at $39^{\circ} \mathrm{C}$ with $5 \% \mathrm{CO}_{2}$ until a few zygotes divided (around $28-30 \mathrm{~h}$ ). Zonae pellucidae were removed by soaking them in a few $\mathrm{mL} 2 \%$ Pronase (Sigma 10165921001) in human tubal fluid (HTF) for less than $2 \mathrm{~min}$ and were then fixed and prepared according to the methods of Simerly and Schatten ${ }^{48}$. In brief, zona-free zygotes were dropped onto a poly-lysine 22-mm square coverslip immersed in protein-free, calcium-free HTF (the coverslip sits in a 6-well dish, with the well full of HTF). We removed most of the HTF using a pipette and replaced it with either $2 \%$ paraformaldehyde in HTF or Buffer M (enough to fill the well), followed by ice-cold methanol (enough to fill the well) for $10-20 \mathrm{~min}$.

Statistical methods. Experiments were repeated at least three times $(N>3)$, and statistical analyses (average $+/$ - standard deviation) were done with Excel. A twotailed, unpaired Student's $t$-test was used to determine $P$-value $(P)$. Chi-squared tests were done as indicated where categorical data was available. ANOVA was done in SPSS with Fisher's LSD post hoc. $P$-value designations are: ${ }^{*} P<0.05$, ${ }^{*} P<$ 0.01 , and ${ }^{* * *} P<0.001$.

Data availability. All data generated or analyzed during this study are included in this published article (and its Supplementary Information files) or are available from the authors.

Received: 9 March 2018 Accepted: 15 May 2018 Published online: 07 June 2018

\section{References}

1. Sathananthan, A. H. et al. The sperm centriole: its inheritance, replication and perpetuation in early human embryos. Human Reprod. 11, 345-356 (1996).

2. Crozet, N., Dahirel, M. \& Chesne, P. Centrosome inheritance in sheep zygotes: centrioles are contributed by the sperm. Microsc. Res. Tech. 49, 445-450 (2000).

3. Palermo, G. D., Colombero, L. T. \& Rosenwaks, Z. The human sperm centrosome is responsible for normal syngamy and early embryonic development. Rev. Reprod. 2, 19-27 (1997).

4. Simerly, C. et al. The paternal inheritance of the centrosome, the cell's microtubule-organizing center, in humans, and the implications for infertility. Nat. Med. 1, 47-52 (1995).

5. Manandhar, G. \& Schatten, G. Centrosome reduction during Rhesus spermiogenesis: gamma-tubulin, centrin, and centriole degeneration. Mol. Reprod. Dev. 56, 502-511 (2000).

6. Manandhar, G., Simerly, C. \& Schatten, G. Highly degenerated distal centrioles in rhesus and human spermatozoa. Human Reprod. 15, 256-263 (2000).

7. Avidor-Reiss, T., Khire, A., Fishman, E. L. \& Jo, K. H. Atypical centrioles during sexual reproduction. Front. Cell Dev. Biol. 3, 21 (2015).

8. Fawcett, D. W. \& Phillips, D. M. The fine structure and development of the neck region of the mammalian spermatozoon. Anat. Rec. 165, 153-164 (1969).

9. Patrick, J., Comizzoli, P. \& Elliott, G. Dry preservation of spermatozoa: considerations for different species. Biopreservation Biobanking 15, 158-168 (2017).

10. Ross, L. \& Normark, B. B. Evolutionary problems in centrosome and centriole biology. J. Evolut. Biol. 28, 995-1004 (2015).

11. Chemes, H. E. in The Centrosome 33-48 (Springer, Heide Schatten, Totowa, NJ, USA 2012).

12. Ounjai, P., Kim, K. D., Lishko, P. V. \& Downing, K. H. Three-dimensional structure of the bovine sperm connecting piece revealed by electron cryotomography. Biol. Reprod. 87, 73 (2012).

13. Debec, A., Sullivan, W. \& Bettencourt-Dias, M. Centrioles: active players or passengers during mitosis? Cell. Mol. Life Sci. 67, 2173-2194 (2010).

14. Blachon, $\mathrm{S}$. et al. A proximal centriole-like structure is present in Drosophila spermatids and can serve as a model to study centriole duplication. Genetics 182, 133-144 (2009).

15. Khire, A. et al. Centriole remodeling during spermiogenesis in Drosophila Curr. Biol. 26, 3183-3189 (2016).

16. Gottardo, M., Callaini, G. \& Riparbelli, M. G. Structural characterization of procentrioles in Drosophila spermatids. Cytoskeleton 72, 576-584 (2015).

17. Khire, A., Vizuet, A. A., Davila, E. \& Avidor-Reiss, T. Asterless reduction during spermiogenesis is regulated by Plk4 and is essential for zygote development in Drosophila. Curr. Biol. 25, 2956-2963 (2015).

18. Baker, M. A. et al. Head and flagella subcompartmental proteomic analysis of human spermatozoa. Proteomics 13, 61-74 (2013).

19. Baker, M. A. et al. Identification of gene products present in Triton X-100 soluble and insoluble fractions of human spermatozoa lysates using LC-MS/ MS analysis. Proteom. Clin. Appl. 1, 524-532 (2007).

20. Amaral, A. et al. Human sperm tail proteome suggests new endogenous metabolic pathways. Mol. Cell. Proteom. 12, 330-342 (2013). 
21. Wang, G. et al. In-depth proteomic analysis of the human sperm reveals complex protein compositions. J. Proteom. 79, 114-122 (2013).

22. Alves-Cruzeiro, J. M., Nogales-Cadenas, R. \& Pascual-Montano, A. D. CentrosomeDB: a new generation of the centrosomal proteins database for human and Drosophila melanogaster. Nucleic Acids Res. 42, D430-D436 (2014).

23. Azimzadeh, J. et al. hPOC5 is a centrin-binding protein required for assembly of full-length centrioles. J. Cell Biol. 185, 101-114 (2009).

24. Craige, B. et al. CEP290 tethers flagellar transition zone microtubules to the membrane and regulates flagellar protein content. J. Cell Biol. 190, 927-940 (2010).

25. Fishman, E. L. et al. Atypical centrioles are present in Tribolium sperm. Open Biol. https://doi.org/10.1098/rsob.160334 (2017).

26. Lattao, R., Kovacs, L. \& Glover, D. M. The centrioles, centrosomes, basal bodies, and cilia of Drosophila melanogaster. Genetics 206, 33-53 (2017).

27. Blachon, S., Khire, A. \& Avidor-Reiss, T. The origin of the second centriole in the zygote of Drosophila melanogaster. Genetics 197, 199-205 (2014).

28. Gonczy, P. Towards a molecular architecture of centriole assembly. Nat. Rev. Mol. Cell Biol. 13, 425-435 (2012).

29. Winey, M. \& O'Toole, E. Centriole structure. Philos. Trans. R. Soc. Lond. B Biol. Sci. https://doi.org/10.1098/rstb.2013.0457 (2014).

30. Chang, C. W., Hsu, W. B., Tsai, J. J., Tang, C. J. \& Tang, T. K. CEP295 interacts with microtubules and is required for centriole elongation. J. Cell Sci. 129, 2501-2513 (2016).

31. Lukinavicius, G. et al. Selective chemical crosslinking reveals a Cep57-Cep63Cep152 centrosomal complex. Curr. Biol. 23, 265-270 (2013).

32. Ibrahim, R., Messaoudi, C., Chichon, F. J., Celati, C. \& Marco, S. Electron tomography study of isolated human centrioles. Microsc. Res. Tech. 72, 42-48 (2009).

33. Paintrand, M., Moudjou, M., Delacroix, H. \& Bornens, M. Centrosome organization and centriole architecture: their sensitivity to divalent cations. J. Struct. Biol. 108, 107-128 (1992).

34. Simerly, C. et al. Biparental inheritance of gamma-tubulin during human fertilization: molecular reconstitution of functional zygotic centrosomes in inseminated human oocytes and in cell-free extracts nucleated by human sperm. Mol. Biol. Cell 10, 2955-2969 (1999).

35. Stearns, T. \& Kirschner, M. In vitro reconstitution of centrosome assembly and function: the central role of gamma-tubulin. Cell 76, 623-637 (1994).

36. Navara, C. S., First, N. L. \& Schatten, G. Microtubule organization in the cow during fertilization, polyspermy, parthenogenesis, and nuclear transfer: the role of the sperm aster. Dev. Biol. 162, 29-40 (1994).

37. Schatten, G. The centrosome and its mode of inheritance: the reduction of the centrosome during gametogenesis and its restoration during fertilization. Dev. Biol. 165, 299-335 (1994).

38. Zernicka-Goetz, M. Cleavage pattern and emerging asymmetry of the mouse embryo. Nat. Rev. Mol. Cell Biol. 6, 919-928 (2005).

39. Bangs, F. K., Schrode, N., Hadjantonakis, A. K. \& Anderson, K. V. Lineage specificity of primary cilia in the mouse embryo. Nat. Cell Biol. 17, 113-122 (2015).

40. Nechipurenko, I. V., Berciu, C., Sengupta, P. \& Nicastro, D. Centriolar remodeling underlies basal body maturation during ciliogenesis in Caenorhabditis elegans. eLife. https://doi.org/10.7554/eLife.25686 (2017).

41. Zebrowski, D. C. et al. Developmental alterations in centrosome integrity contribute to the post-mitotic state of mammalian cardiomyocytes. eLife. https://doi.org/10.7554/eLife.05563 (2015).

42. Dallai, R., Mercati, D., Lino-Neto, J., Dias, G. \& Lupetti, P. Evidence of a procentriole during spermiogenesis in the coccinellid insect Adalia decempunctata (L): an ultrastructural study. Arthropod Struct. Dev. 46, 815-823 (2017).

43. Wong, Y. L. et al. Cell biology. Reversible centriole depletion with an inhibitor of Polo-like kinase 4. Science 348, 1155-1160 (2015).

44. Herbert, M. \& Turnbull, D. Mitochondrial replacement to prevent the transmission of mitochondrial DNA disease. EMBO Rep. 16, 539-540 (2015).

45. Pearson, C. G., Osborn, D. P., Giddings, T. H. Jr., Beales, P. L. \& Winey, M. Basal body stability and ciliogenesis requires the conserved component Poc1. J. Cell Biol. 187, 905-920 (2009).

46. Murray, A. W. \& Kirschner, M. W. Cyclin synthesis drives the early embryonic cell cycle. Nature 339, 275-280 (1989).

47. Hannak, E. \& Heald, R. Investigating mitotic spindle assembly and function in vitro using Xenopus laevis egg extracts. Nat. Protoc. 1, 2305-2314 (2006).

48. Simerly, C. \& Schatten, G. Techniques for localization of specific molecules in oocytes and embryos. Methods Enzymol. 225, 516-553 (1993).

\section{Acknowledgements}

We would like to thank Dotty Sorenson, Sasha Meshinchi, Jeff Harrison, and Chris Edwards for assistance at the Microscopy \& Image Analysis Laboratory in the University of Michigan Medical School; the EPIC facility of Northwestern University's NUANCE Center, which has received support from the Soft and Hybrid Nanotechnology Experimental (SHyNE) Resource (NSF ECCS-1542205); the MRSEC program (NSF DMR$1121262)$ at the Materials Research Center; the International Institute for Nanotechnology (IIN); the Keck Foundation; and the State of Illinois, through the IIN, and Charlene Wilke at the Biological Imaging Facility at Northwestern University. We thank Dr. Richard Komuniecki and Ashtyn Zinn for assistance in preparing the manuscript, Jillian Koffman for assistance with sperm staining, and Dr. Tomohito Higashi and Dr. Elaina Breznau for assistance generating Xenopus egg extracts. We thank the following people for providing antibodies: Dr. Tang K. Tang (CEP135 and CEP120), Dr. Chad Pearson (POC1B), Dr. Andrew Fry (POC1B and POC1A), Dr. Bryan Dynlacht (CEP76), Dr. Jay Gopalakrishnan (CPAP), Dr. Stephen Doxsey (CP110), Dr. Jordan Raff (RTTN), Dr. Erich Nigg (CEP152), Dr. Ingrid Hoffman (CEP152), Dr. Jeremy Reiter (OFD1 and CEP131), Dr. Frans Van Der Hoorn (SPAG4), Dr. Kyung Lee (CEP152), Dr. Michel Bornens (POC5 and CEP89), Dr. Yanagimachi and Dr. Yan (SPATA6), Dr. William Chaney (LGALS3BP), Dr. William Tsang (CEP78), Dr. Jens Luders (Spice1), Dr. František Liška (Centrobin), Dr. Fanni Gergley (CEP63), Dr. Robert Qi (CDK5RAP2), Dr. David Sharp (CEP192), Dr. Gislene Pereira (CEP164), Dr. Joseph Gleeson (CEP41), and Dr. Oliver Gruss (SSX2IP); and Dr. Toshihiko Ezashi and Dr. R. Michael Roberts at the University of Missouri-Columbia for the bovine cell line and Dr. Deborah Chadee for the U2OS cells; and Coba Select Sires for bovine spermatozoa and Scholl Slaughterhouse for bovine testes; and Dr. Steve Pool at Fairfax Cryobank, and Ty Kaliski from Manhattan Cryobank for human sperm. We thank Dr. Barbra Saltzman for statistical advisement, Allen Schroering for testes dissection, embedding, and sectioning, and Gregg Sobocinski at the University of Michigan, and Genevive Phillips and Sarah Crowe from Leica for assistance with STED. This work was supported by grant R01GM098394 from the National Institute of General Medical Sciences and R21HD092700 Eunice Kennedy Shriver National Institute of Child Health and Human Development.

\section{Author contributions}

T.A.-R. supervised the project. E.L.F. led the project. E.L.F., with Q.P.H.N. and V.M., performed the SIM-3D on human sperm; with C.S. and G.S., performed the bovine zygote staining; and with A.L.M., performed the Xenopus egg extract experiments; D.K and J.L. performed correlative light and electron microscopy and with E.L.F., Bovine STORM. K.J. performed electron microscopy. R.R. helped with light microscopy. A.C. helped in developing the POC1B Abs. S.K. performed the U2OS cell staining.

\section{Additional information}

Supplementary Information accompanies this paper at https://doi.org/10.1038/s41467 018-04678-8.

Competing interests: The authors declare no competing interests.

Reprints and permission information is available online at http://npg.nature.com/ reprintsandpermissions/

Publisher's note: Springer Nature remains neutral with regard to jurisdictional claims in published maps and institutional affiliations.

Open Access This article is licensed under a Creative Commons Attribution 4.0 International License, which permits use, sharing, adaptation, distribution and reproduction in any medium or format, as long as you give appropriate credit to the original author(s) and the source, provide a link to the Creative Commons license, and indicate if changes were made. The images or other third party material in this article are included in the article's Creative Commons license, unless indicated otherwise in a credit line to the material. If material is not included in the article's Creative Commons license and your intended use is not permitted by statutory regulation or exceeds the permitted use, you will need to obtain permission directly from the copyright holder. To view a copy of this license, visit http://creativecommons.org/ licenses/by/4.0/

(C) The Author(s) 2018 